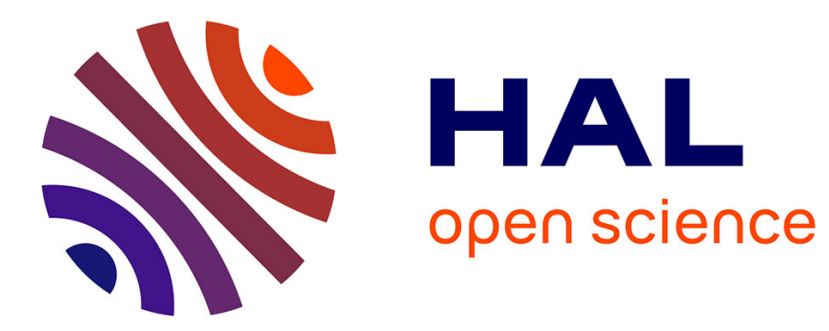

\title{
Rovibrational Energies of the Hydrocarboxyl Radical from a $\operatorname{RCCSD}(T)$ Study
}

\author{
M. Mladenovic
}

\section{To cite this version:}

M. Mladenovic. Rovibrational Energies of the Hydrocarboxyl Radical from a RCCSD(T) Study. Journal of Physical Chemistry A, 2013, 117, pp.7224-7235. 10.1021/jp401151n . hal-00823744

\section{HAL Id: hal-00823744 \\ https://hal.science/hal-00823744}

Submitted on 17 May 2013

HAL is a multi-disciplinary open access archive for the deposit and dissemination of scientific research documents, whether they are published or not. The documents may come from teaching and research institutions in France or abroad, or from public or private research centers.
L'archive ouverte pluridisciplinaire HAL, est destinée au dépôt et à la diffusion de documents scientifiques de niveau recherche, publiés ou non, émanant des établissements d'enseignement et de recherche français ou étrangers, des laboratoires publics ou privés. 


\title{
Rovibrational Energies of the Hydrocarboxyl Radical from a RCCSD(T) Study
}

\author{
Mirjana Mladenović* \\ Université Paris-Est, Laboratoire Modélisation et Simulation Multi Echelle, \\ MSME UMR 8208 CNRS, 5 bd Descartes, 77454 Marne la Vallée, France
}

(Dated: April 2, 2013)

\begin{abstract}
A RCCSD $(\mathrm{T}) / \mathrm{cc}-\mathrm{pVQZ}$ potential energy surface is constructed for the HOCO radical in the ground electronic state and used to compute rotation-vibration levels of HOCO and DOCO. Two numerical strategies are employed to study in detail the wavefunction properties. The importance of stretch-bend coupling, such as $\nu_{4} / \nu_{5}$ and $\nu_{3} / \nu_{4}$, for the internal dynamics is demonstrated. The rotational constants computed for the vibrational ground state of trans and cis conformers are in good agreement with experimental values.
\end{abstract}

Keywords: hydrocarboxyl radical; rovibrational energies; intermode coupling; potential energy surface; ab initio calculation

\section{INTRODUCTION}

The reaction between hydroxyl radical and carbon monoxide is known to proceed via an energized HOCO intermediate,

$$
\mathrm{HO}+\mathrm{CO} \rightleftharpoons \mathrm{HOCO} \rightleftharpoons \mathrm{H}+\mathrm{CO}_{2} .
$$

This was first proposed by Smith and Zellner ${ }^{1,2}$ and later verified in photoionization, ${ }^{3}$ ultrashort laser pulse, ${ }^{4}$ and infrared spectroscopic studies. ${ }^{5,6}$ The process of Eq. (1) provides the most common pathway for atmospheric depletion of both $\mathrm{OH}$ and $\mathrm{CO}$ and is the principal source of heat in hydrocarbon flames. ${ }^{7,8}$ It is also known that the reaction of Eq. (1) exhibits a significant non-statistical (non-Arrhenius) behaviour. ${ }^{9-12}$

The stability of the HOCO radical was first observed in a low-temperature CO matrix isolation study of Milligan and Jacox. ${ }^{13}$ These authors showed the existence of two geometric isomers, trans- and cis-HOCO, and assigned five fundamental transitions. Later studies in an Ar matrix ${ }^{14}$ concluded that trans-HOCO is more stable than cis-HOCO. The first gas phase detection of HOCO is due to Ruscic et $a l,{ }^{3}$ who found in a photoionization mass spectrometric study that HOCO is bound by 10.2 $\mathrm{kcal} / \mathrm{mol}\left(3600 \mathrm{~cm}^{-1}\right)$ with respect to the lowest energy dissociation products, $\mathrm{H}+\mathrm{CO}_{2}$.

Experimental spectroscopic parameters of $\mathrm{HOCO}$ are still scarce in the literature. High resolution gas phase data are available for the vibrational ground state, ${ }^{15-17}$ the fundamental $\nu_{2}(\mathrm{C}=\mathrm{O}$ stretching $)$ vibration, ${ }^{18}$ and the fundamental $\nu_{1}$ (H-O stretching) vibration ${ }^{19,20}$ of trans-HOCO and trans-DOCO. The first high-resolution spectra for cis-HOCO and cis-DOCO were obtained only recently. By means of Fourier transform microwave spectroscopy, Oyama et al. ${ }^{21}$ recorded in total 25 lines for cis-HOCO and 46 lines for cis-DOCO, both in the vibrational ground state, and derived spectroscopic parameters of high accuracy. Recent photoelectron spectra due to Johnson et al..$^{22}$ provide the first gas phase observation of the low-frequency in-plane modes for both conformers of HOCO and DOCO.

In the theoretical front, a great deal of work has been focused on the curious kinetic behaviour of the reaction of Eq. (1). ${ }^{12}$ Several global potential energy surfaces were constructed for reaction dynamics investigations. ${ }^{23-27}$ The potential energy surface of Schatz, Fitzcharles, and Harding, ${ }^{23}$ which went through several iterations, ${ }^{25,28,29}$ was used also for bound state calculations carried out by means of a five-dimensional approch, ${ }^{30}$ a MULTIMODE approach, ${ }^{31}$, and by numerically exact full-dimenional strategies. ${ }^{32,33}$ Botschwina ${ }^{34}$ calculated the equilibrium structures of both trans-HOCO and cis-HOCO at the level of the spin restricted coupled cluster theory and made predictions for the ground-state rotational constants of cis-HOCO that agree well with the experimental results published six years later. ${ }^{21}$ Spectroscopic properties of HOCO were studied in detail by Fortenberry et al. who recently developed separate quartic force fields for trans-HOCO [Ref. 35] and for cis-HOCO [Ref. 36], which were used to compute the fundamental frequencies, ground-state rotational constants, and centrifugal distortion constants by means of vibrational second order perturbation theory and vibrational configuration interaction. These force fields were reused in combination with a 6181-term RCCSD/aug-cc-pVTZ dipole moment surface to compute MULTIMODE infrared intensities for vibrational transitions of less than $4000 \mathrm{~cm}^{-1} .{ }^{37}$ Wang et al. ${ }^{38}$ presented results for some vibrational transitions up to $2 \nu_{1}$ and their intensities, obtained by means of three numerical approaches from a 918-term UCCSD(T)-F12/aug-cc-pVTZ potential energy surface common for the two HOCO forms and a 1928-term UCCSD(T)-F12/aug-cc-pVDZ dipole moment surface.

Our own interest in $\mathrm{HOCO}$ arises from the new experimental information on the pure rotational spectra ${ }^{21}$ and the low-frequency modes. ${ }^{22}$ The primary theoretical interest was awakened by the existence of two stable isomers undergoing large-amplitude torsional motion. For the electronic ground state $\mathrm{X}^{2} A^{\prime}$, we developed a new potential energy surface (PES) based on spin restricted coupled cluster calculations (Section II). The PES covers both isomers in the range of spectroscopic interest and allows consideration of large amplitude torsional motion. Vibrational and rovibrational calculations carried out by 
means of two computational methodologies (Section III) are used to derive the fundamental transitions (Section IV A), the torsional levels (Section IV B), and the effective rotational constants for the vibrational ground state of the trans and cis forms of HOCO and DOCO (Section IV C).

\section{POTENTIAL ENERGY SURFACE}

The six-dimensional (6D) potential energy surface for the electronic ground state $\left(\mathrm{X}^{2} A^{\prime}\right)$ of $\mathrm{HOCO}$ was scanned by means of the partially spin adapted coupled cluster RCCSD(T) method including single and double excitations and perturbative corrections for triple excitations. ${ }^{39-41}$ The correlation consistent quadruple zeta basis set, commonly abbreviated as cc-pVQZ, was used. Only valence electrons were correlated. All calculations were carried out with the MOLPRO quantum chemistry program package. ${ }^{42}$

The coordinate space was parametrized in terms of the bond-distance-bond-angle coordinates $r_{1}, r_{2}, r_{3}, \alpha$, $\beta$, and $\tau$. The three bond distances are $r_{1}=r(\mathrm{H}-\mathrm{O})$, $r_{2}=r(\mathrm{O}-\mathrm{C})$, and $r_{3}=r(\mathrm{C}=\mathrm{O})$. The angles $\alpha$ and $\beta$ stand for the two in-plane bending angles $\angle \mathrm{HOC}$ and $\angle \mathrm{OCO}$, respectively, whereas $\tau$ denotes the dihedral angle measured from the cis side.

The $a b$ initio points were fitted to a six-dimensional analytical expression of the form

$$
\begin{aligned}
V\left(r_{1}, r_{2}, r_{3}, \alpha, \beta, \tau\right)= & \sum_{i, j, k, l, m, n} C_{i j k l m n} s_{1}^{i} s_{2}^{j} s_{3}^{k} \\
& \times\left(\alpha-\alpha_{\mathrm{ref}}\right)^{l}\left(\beta-\beta_{\mathrm{ref}}\right)^{m} \cos (n \tau),
\end{aligned}
$$

where $s_{i}$ for $i=1,2,3$ stands for the modified Morse coordinates, ${ }^{43}$

$$
s_{i}=\frac{1}{a_{i}}\left[1-e^{-a_{i}\left(r_{i} / r_{i}^{\mathrm{ref}}-1\right)}\right] .
$$

The geometry of the torsional saddle point was chosen to define the reference geometry $\left\{r_{1}^{\text {ref }}, r_{2}^{\text {ref }}, r_{3}^{\text {ref }}, \alpha_{\text {ref }}, \beta_{\text {ref }}\right\}$ in Eqs. (2) and (3). Optimum values for the three nonlinear parameters $a_{i}$ of the Morse coordinates $s_{i}$ from Eq. (3) were obtained with a Levenberg-Marquardt nonlinear least-squares algorithm. ${ }^{44}$ For given $a_{i}$, the expansion parameteres $C_{i j k l m n}$ were determined by means of a linear least-squares procedure.

In total, $3261 \mathrm{RCCSD}(\mathrm{T}) / \mathrm{cc}-\mathrm{pVQZ}$ points with energies up to $10000 \mathrm{~cm}^{-1}$ above the trans minimum were considered. Some of the $a b$ initio points previously calculated were also used. ${ }^{45}$ For the final fitting, a weight of a $i$ th data point of energy $E_{i}$ was set to $1 /\left(E_{i}+2500 \mathrm{~cm}^{-1}\right)$, as previously done in our study of $\mathrm{HCCN}^{-}$[Ref. 46]. All parameters smaller than one and two times their standard deviation and most of the parameters smaller than three times their standard deviation were eliminated in repeated fitting cycles. In this fashion, we arrived at a functional six-dimensional expansion consisting of 191 terms. The standard deviation of the weighted 191term expansion was $2.5 \mathrm{~cm}^{-1}$. The expansion coefficients $C_{i j k l m n}$ of this potential energy representation are summarized in Table I. The zero of the energy scale is defined as the energy of the trans minimum.

\section{A. Topography of the potential energy surface}

The structural parametres of trans-HOCO, cis-HOCO and the torsional saddle point $\tau$-HOCO obtained for the present RCCSD $(\mathrm{T}) / \mathrm{cc}-\mathrm{pCVQZ}$ potential energy surface are summarized in Table II. There we also list all electron RCCSD $(\mathrm{T}) / \mathrm{cc}-\mathrm{pCVQZ}$ results due to Botschwina ${ }^{34}$ and RCCSD $(\mathrm{T}) / \mathrm{CBS}$ results due to Fortenberry et al. ${ }^{35,36}$ obtained at the estimated complete basis set (CBS) limit. As seen in Table II, the optimum bond lengths and the optimum bond angles all agree within $0.009 \mathrm{a}_{0}$ and $0.3^{\circ}$, respectively. The equilibrium rotational constants $A_{e}$ and $B_{e}, C_{e}$ show agreement within respectively 0.04 and $0.002 \mathrm{~cm}^{-1}$, i.e. within $0.6 \%$.

The two-dimensional contour maps of the sixdimensional RCCSD(T)/cc-pVQZ PES are displayed in Fig. 1. The contour maps clearly show that HOCO is a planar molecule possessing trans and cis minima. The cis minimum is $603 \mathrm{~cm}^{-1}$ above the trans counterpart, as seen in Table II. The torsional saddle point at $\tau=86.7^{\circ}$ is $3270 \mathrm{~cm}^{-1}$ above the trans form (Table II). The minimum energy path (MEP) along the torsional coordinate is obtained by energy minimization with respect to the other five coordinates. The optimum bond distance $r_{2}^{o p t}$ and the optimum bond angles $\alpha_{o p t}$ and $\beta_{o p t}$ exhibit only weak torsional dependences (within $0.06 \mathrm{a}_{0}$ and $3^{\circ}$ ) along the torsional minimum energy path in Fig. 1. For the optimum $r_{1}$ and $r_{3}$ values, we found variations within 0.01 $\mathrm{a}_{0}$. This finding readily indicates only weak coupling between the torsion and the in-plane vibrational modes.

\section{BOUND STATE CALCULATIONS}

In the bound state calculations, we employed the orthogonal (diatom+diatom) coordinates $d_{1}, d_{2}, R, \theta_{1}, \theta_{2}, \chi$ and the body-fixed expression of the kinetic energy operator. ${ }^{33}$ For $\mathrm{HO}^{a} \mathrm{CO}^{b}$, the coordinates $d_{1}$ and $d_{2}$ refer to the $\mathrm{H}_{-} \mathrm{O}^{a}$ and $\mathrm{C}-\mathrm{O}^{b}$ bond lengths, $R$ is the distance between the centers of mass of the $\mathrm{HO}^{a}$ and $\mathrm{CO}^{b}$ subunits, $\theta_{i}$ is the angle enclosed by the vectors $\mathbf{d}_{i}$ and $\mathbf{R}$ $(i=1,2)$, and $\chi$ is the out-of-plane (torsional) angle. The body-fixed $z$ axis is chosen to be aligned with the vector R. ${ }^{33}$ The (diatom+diatom) coordinates are particularly well suited to describe the dissociation of HOCO into the channel $\mathrm{HO}+\mathrm{CO}$.

For the rovibrational calculations, two numerical approaches were used, both employing a discrete variable representation (DVR) and a parity-adapted rotationalangular basis set. These methods involve no dynamical approximations and no re-expansion of the potential en- 
TABLE I: Expansion coefficients $C_{i j k l m n}$ (in atomic units) of Eq. (2) for the six-dimensional RCCSD(T)/cc-pVQZ potential energy surface developed in the present work for the ground electronic state of HOCO. The reference geometry is given by $r_{1}^{\text {ref }}=1.82357 a_{0}, r_{2}^{\text {ref }}=2.57782 a_{0}, r_{3}^{\text {ref }}=2.22459 a_{0}, \alpha_{\text {ref }}=108.623^{\circ}$, and $\beta_{\text {ref }}=129.433^{\circ}$. The exponential parameters $a_{1}=0.4026, a_{2}=0.8738$, and $a_{3}=1.6075$ are used for the Morse coordinates $s_{1}, s_{2}$, and $s_{3}$ of Eq. (3), respectively.

\begin{tabular}{|c|c|c|c|c|c|c|c|}
\hline$i j k l m n$ & $C_{i j k l m n}$ & $i j k l m n$ & $C_{i j k l m n}$ & $i j k l m n$ & $C_{i j k l m n}$ & $i j k l m n$ & $C_{i j k l m n}$ \\
\hline 000000 & 0.00836781 & 100001 & -0.00745361 & 000101 & -0.00126955 & 001101 & -0.00725039 \\
\hline 100000 & -0.00055379 & 100002 & -0.00087982 & 000102 & 0.00279844 & 001102 & 0.00257248 \\
\hline 200000 & 0.85755655 & 100003 & 0.00003830 & 000103 & 0.00069418 & 001110 & -0.05265608 \\
\hline 300000 & -1.59305128 & 200001 & -0.00841369 & 000201 & -0.00043392 & 001111 & 0.02151180 \\
\hline 400000 & 1.88261547 & 200002 & -0.00390640 & 000202 & 0.00707674 & 010011 & 0.00608270 \\
\hline 500000 & -1.92454109 & 300001 & -0.01426951 & 000203 & -0.00044319 & 010012 & -0.00818092 \\
\hline 600000 & 1.44273034 & 011000 & 0.51319721 & 000204 & -0.00036894 & 010021 & -0.08304211 \\
\hline 700000 & -0.67158588 & 012000 & -0.29725722 & 000301 & -0.00090028 & 010101 & -0.00241730 \\
\hline 010000 & 0.02468718 & 021000 & -0.08194301 & 000302 & -0.00160687 & 010102 & -0.00570724 \\
\hline 020000 & 1.07225123 & 010100 & 0.12523030 & 000401 & 0.00446180 & 010103 & -0.00529202 \\
\hline 030000 & -2.90735982 & 010200 & -0.07700392 & 000402 & -0.00228362 & 010110 & -0.01036017 \\
\hline 040000 & 1.72442690 & 010300 & 0.04800357 & 000501 & -0.00295305 & 010120 & 0.36342201 \\
\hline 050000 & 1.55371903 & 020100 & -0.13062138 & 000011 & -0.00943983 & 010130 & -0.50925160 \\
\hline 060000 & -1.38833909 & 020200 & -0.07501467 & 000012 & 0.00277347 & 010201 & -0.00662024 \\
\hline 001000 & -0.00119757 & 020300 & 0.13649482 & 000013 & 0.00049530 & 010202 & -0.02211013 \\
\hline 002000 & 2.32154526 & 030100 & -0.31949891 & 000014 & 0.00019359 & 010301 & 0.01981882 \\
\hline 003000 & -2.72709885 & 030200 & 0.18828323 & 000021 & 0.00273316 & 011001 & -0.00248425 \\
\hline 004000 & 1.05170410 & 010010 & 0.11764941 & 000022 & -0.00427681 & 011002 & -0.00683282 \\
\hline 005000 & -0.57951955 & 010020 & -0.25158541 & 000031 & -0.00502948 & 011010 & -0.50042611 \\
\hline 000100 & 0.00296966 & 020010 & -0.20297505 & 000032 & 0.00217071 & 011011 & -0.02847034 \\
\hline 000200 & 0.07610313 & 010001 & 0.00636039 & 111000 & 0.04613353 & 011100 & 0.00732831 \\
\hline 000300 & -0.03567142 & 010002 & 0.02414256 & 000111 & -0.03646118 & 020011 & 0.17384948 \\
\hline 000400 & -0.00610234 & 010003 & 0.00024572 & 000112 & -0.00228122 & 020101 & 0.02813291 \\
\hline 000500 & -0.00156040 & 010004 & -0.00093246 & 000113 & 0.00141805 & 020110 & 0.56411952 \\
\hline 000600 & 0.00406369 & 020001 & -0.00364918 & 000121 & -0.00872518 & 020111 & 0.62953502 \\
\hline 000700 & -0.00244732 & 020002 & -0.01526453 & 000122 & 0.00186631 & 030011 & -0.43577789 \\
\hline 000800 & 0.00083233 & 030001 & -0.01473643 & 000131 & 0.02191070 & 100011 & -0.02483552 \\
\hline 000010 & 0.00309784 & 030002 & -0.05900073 & 000132 & 0.00828586 & 100012 & 0.00100967 \\
\hline 000020 & 0.15477242 & 040001 & 0.00806554 & 000141 & -0.04358681 & 100021 & -0.00884712 \\
\hline 000030 & -0.05398365 & 001100 & 0.00157014 & 000142 & -0.01440554 & 100101 & -0.00192780 \\
\hline 000040 & 0.03131505 & 001200 & -0.01267875 & 000151 & 0.03819373 & 100102 & -0.00381710 \\
\hline 000050 & -0.02190456 & 002100 & -0.02414472 & 000211 & 0.01021089 & 100103 & 0.00123519 \\
\hline 000060 & -0.00321959 & 001010 & 0.08973371 & 000212 & -0.00142220 & 100211 & -0.10404632 \\
\hline 000001 & 0.00146993 & 001020 & -0.24588891 & 000221 & 0.01273082 & 100301 & 0.03558735 \\
\hline 000002 & -0.00635973 & 001001 & -0.01066330 & 000222 & 0.01064010 & 101001 & 0.01065118 \\
\hline 000003 & -0.00003421 & 001002 & -0.00175187 & 000231 & -0.02983697 & 101002 & 0.00174893 \\
\hline 000004 & 0.00012628 & 000110 & 0.00037507 & 000232 & -0.01651322 & 101011 & 0.05226679 \\
\hline 000005 & 0.00000067 & 000120 & -0.00381365 & 000241 & 0.02951766 & 110001 & 0.03326665 \\
\hline 110000 & 0.01766263 & 000130 & 0.00488718 & 000242 & 0.02458448 & 110002 & 0.00934577 \\
\hline 120000 & -0.17420096 & 000140 & -0.01395447 & 000311 & 0.01831219 & 110010 & 0.06755521 \\
\hline 210000 & 0.07719126 & 000210 & -0.01364521 & 000321 & -0.00418284 & 110011 & 0.13688878 \\
\hline 101000 & -0.01366984 & 000220 & 0.01794977 & 000322 & -0.00446691 & 110100 & -0.15156445 \\
\hline 102000 & -0.08398039 & 000230 & -0.01764837 & 000411 & 0.00852331 & 110200 & 0.50257917 \\
\hline 100100 & 0.03938215 & 000310 & 0.00692572 & 000412 & -0.00157593 & 110201 & -0.14569645 \\
\hline 100200 & -0.04843377 & 000320 & 0.01228029 & 000511 & -0.00572459 & 111001 & -0.03212341 \\
\hline 100300 & 0.06579241 & 000420 & -0.01487149 & 000312 & 0.00302460 & 120001 & -0.03249419 \\
\hline 200100 & -0.01110065 & 000510 & -0.00174238 & 001011 & 0.01606472 & 210001 & 0.04966861 \\
\hline 100010 & -0.01133534 & 000610 & 0.00202162 & 001012 & 0.00271731 & & \\
\hline
\end{tabular}


TABLE II: Geometric parameters of trans-HOCO, cis-HOCO, and the torsional saddle point $\tau$-HOCO obtained for the RCCSD $(\mathrm{T}) / \mathrm{cc}$-pVQZ potential energy surface developed here and in previous theoretical studies. ${ }^{34-36}$ The energy measured relative to the energy of the trans minimum is denoted by $\mathrm{E}_{r e l}$. The equilibrium rotational constants $A_{e}, B_{e}$, and $C_{e}$ are additionally shown.

\begin{tabular}{|c|c|c|c|c|c|c|c|}
\hline & \multicolumn{3}{|c|}{ trans-HOCO } & \multicolumn{3}{|c|}{ cis-HOCO } & \multirow{2}{*}{$\frac{\tau \text {-HOCO }}{\text { This work }}$} \\
\hline & This work & Ref. 34 & Ref. 35 & This work & Ref. 34 & Ref. 36 & \\
\hline$\tau / \operatorname{deg}$ & 180.0 & 180.0 & 180.0 & 0.0 & 0.0 & 0.0 & 86.72 \\
\hline$\alpha / \operatorname{deg}$ & 107.68 & 107.81 & 107.99 & 107.93 & 108.07 & 108.21 & 108.63 \\
\hline$\beta / \operatorname{deg}$ & 127.03 & 127.11 & 126.98 & 130.27 & 130.33 & 130.24 & 129.45 \\
\hline$r_{1} / a_{0}$ & 1.8186 & 1.8174 & 1.8165 & 1.8364 & 1.8351 & 1.8348 & 1.8237 \\
\hline$r_{2} / a_{0}$ & 2.5388 & 2.5324 & 2.5307 & 2.5144 & 2.5086 & 2.5057 & 2.5776 \\
\hline$r_{3} / a_{0}$ & 2.2266 & 2.2223 & 2.2208 & 2.2364 & 2.2320 & 2.2306 & 2.2246 \\
\hline $\mathrm{E}_{r e l} / \mathrm{cm}^{-1}$ & 0.0 & 0.0 & & 603.3 & 610.8 & & 3269.9 \\
\hline $\mathrm{A}_{e} / \mathrm{cm}^{-1}$ & 5.558 & 5.596 & 5.586 & 4.723 & 4.739 & 4.733 & 4.961 \\
\hline $\mathrm{B}_{e} / \mathrm{cm}^{-1}$ & 0.383 & 0.384 & 0.385 & 0.393 & 0.395 & 0.395 & 0.373 \\
\hline $\mathrm{C}_{e} / \mathrm{cm}^{-1}$ & 0.358 & 0.359 & 0.360 & 0.363 & 0.364 & 0.365 & 0.358 \\
\hline
\end{tabular}
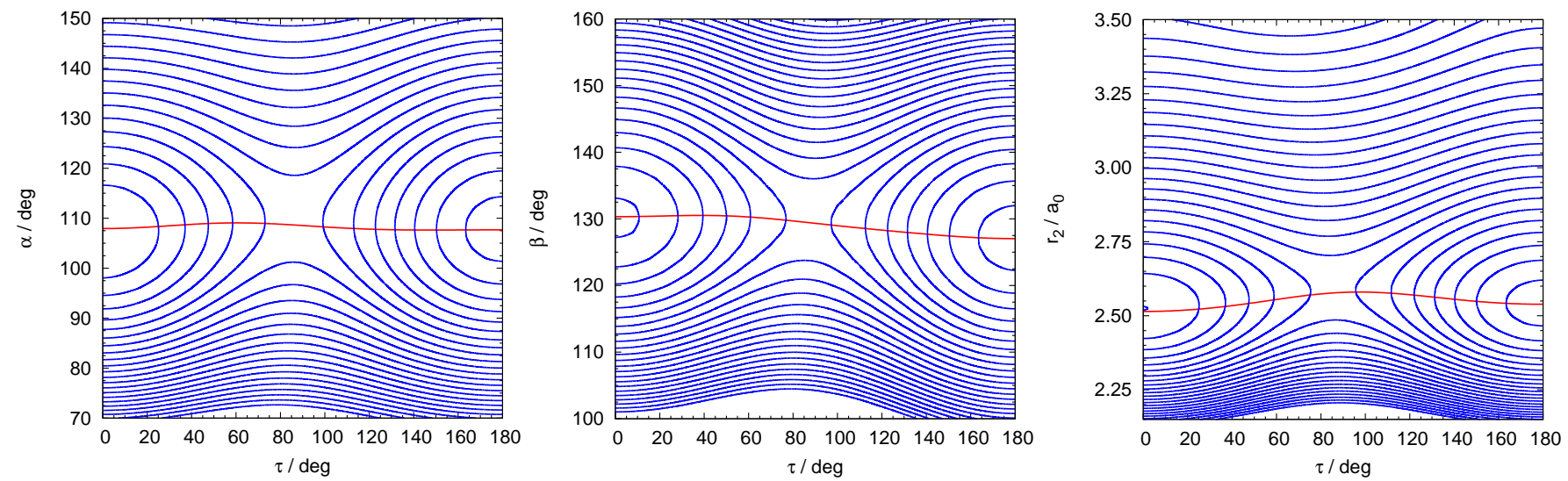

FIG. 1: Two-dimensional $(\tau, \alpha),(\tau, \beta)$, and $\left(\tau, r_{2}\right)$ contour maps of the six-dimensional RCCSD(T) PES. The remaining four coordinates are kept constant at their equilibrium values for the trans isomer. Contour lines are drawn in intervals of $500 \mathrm{~cm}^{-1}$ with the first contour placed at $250 \mathrm{~cm}^{-1}$. The solid (red online) line gives the variation of the internal coordinate shown on the $y$ axis along the torsional minimum energy path. 
ergy. The DVR(6) method ${ }^{47,48}$ is based on the discretization of the entire six-dimensional angular-radial space. The $\operatorname{DVR}(6)$ calculations were carried out for $J=0$ by means of primary or/and potential optimized DVR points. The second set of calculations was performed with the help of the DVR $(+\mathrm{R})+\mathrm{FBR}$ approach, ${ }^{46-48}$ using combined discrete variable and finite basis representations for the angular coordinates $\left(\theta_{1}, \theta_{2}, \chi\right)$ in combination with a discretized Jacobi distance $R$, eigenfunctions for $\left(d_{1}, d_{2}\right)$, and symmetric top eigenfunctions for the rotational part. The DVR $(6)$ and $\operatorname{DVR}(+\mathrm{R})+\mathrm{FBR}$ methods both employ a contraction scheme resulting from several diagonalization/truncation steps to construct a compact basis set. The final matrix of modest size is diagonalized by standard routines, providing energies and wave functions in a simple fashion.

The DVR $(6)$ and $\operatorname{DVR}(+\mathrm{R})+\mathrm{FBR}$ approaches make use of several adiabatic projection schemes to study separability of (ro)vibrational motions (intermode couplings/mixing) and to characterize the exact quantum states. The computational methodology of the $\operatorname{DVR}(6)$ approach readily permits the construction of the adiabatic (zero-order) basis, consisting of eigenvectors computed in adiabatic torsion and in adiabatic bend approximations. The DVR $(+\mathrm{R})+\mathrm{FBR}$, on the other hand, allows easy determination of the zero-order base in the adiabatic stretch $(R)$ approximation. The quantum state character correlation scheme ${ }^{47}$ is employed to monitor the evolution of the adiabatic representation in both $\operatorname{DVR}(6)$ and $\operatorname{DVR}(+\mathrm{R})+\mathrm{FBR}$ computational approaches.

The DVR(6) and DVR $(+\mathrm{R})+\mathrm{FBR}$ approaches use different angular basis sets (of product and nonproduct type, respectively) and consider the contributions to the vibrational Hamiltonian in different order (different diagonalization/truncation schemes). Due to these numerical/algorithmic differences, the two approaches provide complementary information, useful for gaining a deeper insight into the nature of internal molecular dynamics.

\section{A. Numerical aspects}

The DVR(6) calculations for $J=0$ were performed using 20 potential optimized DVR points for each of the two bending angles $\left(\theta_{1}, \theta_{2}\right),[13,9,8]$ potential adapted discrete points for the radial coordinates $\left[R, d_{1}, d_{2}\right]$, and 21 (20) primary DVR points in even (odd) parity for the torsional angle $\chi$, thus, giving the primary base of 7862400 and 7488000 functions in the inversion symmetric and antisymmetric block, respectively. The potential adapted DVR points were determined for the respective reference potentials defined by the equilibrium geometry of cisHOCO. Dimensions of matrices diagonalized at different steps of calculation were in range 1000-2000. The final matrix had a dimension of 6300 and 6000 in even and odd parity, respectively.

In the $\mathrm{DVR}(+\mathrm{R})+\mathrm{FBR}$ calculations, we employed 10 potential optimized DVR points for $R$ and 12 twodimensional eigenfunctions for $d_{1}, d_{2}$, constructed for the trans-HOCO reference geometry. The maximum value $k^{\max }$ for the projection quantum number $k$ of the vibrational angular momentum was 15 in $J=0$ and 18 in $J=4$ calculations. The integrals over the torsional angle were solved by Gauss-Chebyshev quadrature of order 31 . Gauss-Legendre DVR points employed for the bending angles were determined from $P_{l_{i}^{\max }}^{k}$ of $k=0,1, \cdots, k^{\max }$ with $l_{1}^{\max }=30$ for $\theta_{1}$ and $l_{2}^{\max }=60$ for $\theta_{2}$, keeping only the points distributed between $50^{\circ}$ and $180^{\circ}$ for $\theta_{1}$ and between $0^{\circ}$ and $70^{\circ}$ for $\theta_{2}$. The primary basis included 646680 functions for $J=0$ and 5800440 for $J=4$.

The DVR(6) and DVR $(+\mathrm{R})+\mathrm{FBR}$ results show excellent agreement: the fundamental $\nu_{2}, \nu_{3}, \nu_{4}, \nu_{5}, \nu_{6}$ transitions agree better than $0.04 \mathrm{~cm}^{-1}$, whereas we found an agreement within 0.09 and $0.5 \mathrm{~cm}^{-1}$ for $\nu_{1}$ of transHOCO $\left[n^{(0,0)}=99\right]$ and of cis-HOCO $\left[n^{(0,0)}=133\right]$.

\section{RESULTS}

The (ro)vibrational levels of $\mathrm{H} / \mathrm{DOCO}$ were analysed in detail by means of several adiabatic projection schemes, vibrationally averaged geometries, and a visual inspection of wavefunction nodal patterns. The different approaches helped to extract valuable informations on wavefunction properties.

The full-dimensional rovibrational energies are denoted by $E^{(J, p)}$ and the corresponding ordinal numbers by $n^{(J, p)}$ for a given total rotational angular momentum $J$ and parity $p$. As usual, $K$ stands for the quantum number for the body-fixed $z$-projection of $J$. Note that the direction of the diatom-diatom vector $\mathbf{R}$ is found to depart by at most $7^{\circ}$ from the true principal axes of $\mathrm{H} / \mathrm{DOCO}$ along the torsional minimum energy path, such that $\mathbf{R}$ provides a good approximation for the principal moment of inertia axis.

\section{A. Vibrational transitions}

The fundamental vibrational transitions calculated for the present RCCSD $(\mathrm{T}) / \mathrm{cc}-\mathrm{pVQZ}$ PES are compared in Table III with available experimental data and recent theoretical results. The high resolution gas phase data, ${ }^{18-20}$ only available for $\nu_{1}$ and $\nu_{2}$ of trans-HOCO and trans-DOCO, are listed there along with the values for $\nu_{3}, \nu_{4}, \nu_{5}$ of HOCO and for $\nu_{3}, \nu_{5}$ of DOCO from photoelectron spectroscopic studies. ${ }^{22}$ Note that the photoelectron spectroscopic data were reported with uncertainties of $\pm 5 \mathrm{~cm}^{-1}$ for $\nu_{5}$ and $\pm 10 \mathrm{~cm}^{-1}$ for $\nu_{3}$ and $\nu_{4}$. For $\nu_{1}, \nu_{2}$, and $\nu_{6}$ of the cis isomers, the experimental data are available only from matrix isolation studies. ${ }^{13,14,49,50}$ The theoretical results due to Johnson et al. ${ }^{22}$ were obtained by means of second order vibrational perturbation theory (VPT). Since the transitions from the vibrational configuration interaction calculations of Fortenberry et 
TABLE III: Fundamental vibrational transitions of HOCO and DOCO (in $\mathrm{cm}^{-1}$ ) obtained in the present work, in earlier theoretical studies, ${ }^{22,35,36}$ and in experimental studies. ${ }^{13,18-20,22,49,50}$ The experimental results are given in the columns denoted by Expt.

\begin{tabular}{|c|c|c|c|c|c|c|c|c|c|c|c|c|}
\hline & \multicolumn{3}{|c|}{ trans-HOCO } & \multicolumn{3}{|c|}{ cis-HOCO } & \multicolumn{3}{|c|}{ trans-DOCO } & \multicolumn{3}{|c|}{ cis-DOCO } \\
\hline & This work & Ref. 35 & Expt. & This work & Ref. 36 & Expt. & This work & Ref. 22 & Expt. & This work & Ref. 22 & Expt. \\
\hline$\nu_{1}$ & 3648 & 3642 & $3636^{a}$ & 3461 & 3451 & $3411^{e}$ & 2691 & 2688 & $2684^{f}$ & 2558 & 2555 & $2522^{e}$ \\
\hline$\nu_{2}$ & 1863 & 1861 & $1853^{b}$ & 1825 & 1823 & $1802^{e}$ & 1859 & 1845 & $1852^{b}$ & 1829 & 1814 & $1804^{e}$ \\
\hline$\nu_{3}$ & 1217 & 1217 & $1194^{c}$ & 1278 & 1284 & $1290^{c}$ & 1092 & 1081 & $1081^{c}$ & 1132 & 1121 & $1145^{c}$ \\
\hline$\nu_{4}$ & 1061 & 1053 & $1048^{c}$ & 1059 & 1046 & $1040^{c}$ & 906 & 900 & & 961 & 949 & \\
\hline$\nu_{5}$ & 617 & 617 & $629^{c}$ & 600 & 602 & $605^{c}$ & 593 & 588 & $597^{c}$ & 539 & 535 & $557^{c}$ \\
\hline$\nu_{6}$ & 507 & 501 & $508^{d}$ & 543 & 566 & & 396 & 395 & $472^{g}$ & 452 & 454 & $497^{g}$ \\
\hline
\end{tabular}

${ }^{a}$ Reference 19. ${ }^{b}$ Reference 18. ${ }^{c}$ Reference 22. ${ }^{d}$ Reference 49. ${ }^{e}$ Reference 50. ${ }^{f}$ Reference $20 .{ }^{g}$ Reference 13.

$a l^{35,36}$ were found to be less trustworthy compared to the VPT findings, ${ }^{51}$ we give their VPT results in Table III.

\section{Comparison with previous theoretical and experimental work}

Our results for trans-HOCO and for $\nu_{1}, \nu_{2}, \nu_{3}, \nu_{5}$ of cis-HOCO in TableIII agree within $10 \mathrm{~cm}^{-1}$ with the theoretical VPT values of Fortenberry and others; ${ }^{35,36}$ for $\nu_{4}$ and $\nu_{6}$ of cis-HOCO we see deviations of 13 and $24 \mathrm{~cm}^{-1}$, respectively. The agreement with the results of Wang et al. ${ }^{38}$ is within $10 \mathrm{~cm}^{-1}$, except for $\nu_{1}$ and $\nu_{4}$ of cis-HOCO, where the deviations are roughly $20 \mathrm{~cm}^{-1}$. Comparison for DOCO with the theoretical predictions of Johnson et al. ${ }^{22}$ shows agreement within $15 \mathrm{~cm}^{-1}$. For the quartic force fields of Fortenberry et al. we computed the fundamental $\left[\nu_{1}, \nu_{2}, \nu_{3}, \nu_{4}, \nu_{5}, \nu_{6}\right]$ transitions (in $\mathrm{cm}^{-1}$ ) of $[2690,1859,1088,905,592,392]$ for trans-DOCO and [2551, 1827, 1123, 965, 541,467] for cis-DOCO, which agree with our values within $9 \mathrm{~cm}^{-1}$, except for $\nu_{6}$ of cis-DOCO, where we see a difference of $15 \mathrm{~cm}^{-1}$.

The torsional frequencies obtained here are now compared in more detail with previous theoretical values. The harmonic torsional frequencies $\omega_{6}$ for the RCCSD(T)/cc-pVQZ PES, which amount to 535 and 576 $\mathrm{cm}^{-1}$ for respectively trans-HOCO and cis-HOCO, are in excellent agreement with $\omega_{6}$ of 536 and $578 \mathrm{~cm}^{-1}$ reported by Johnson et al..$^{22}$ or 537 and $578 \mathrm{~cm}^{-1}$ found by Fortenberry and others. ${ }^{35,36}$ Our anharmonic $\nu_{6}$ frequencies agree within $2 \mathrm{~cm}^{-1}$ with the VPT results of Johnson et al..$^{22}$ for both isomers of HOCO and DOCO and within $10 \mathrm{~cm}^{-1}$ with the results of Wang et al. ${ }^{38}$ for the HOCO isomers. The agreement with the VPT results of Fortenberry et al..$^{35,36}$ is within $6 \mathrm{~cm}^{-1}$ for trans-HOCO and $23 \mathrm{~cm}^{-1}$ for cis-HOCO. We may, however, note that the VCI torsional transitions at 488.6 for trans-HOCO [Ref. 35] and at $540.2 \mathrm{~cm}^{-1}$ for cis-HOCO [Ref. 36] are smaller by respectively 13 and $26 \mathrm{~cm}^{-1}$ than the corresponding VPT counterparts. The same authors ${ }^{37}$ recently reported a corrected VCI $\nu_{6}$ value of $475 \mathrm{~cm}^{-1}$ for trans-HOCO, along with $\nu_{6}$ of $368 \mathrm{~cm}^{-1}$ for trans-DOCO and $447 \mathrm{~cm}^{-1}$ for cis-DOCO. These VCI results for $\nu_{6}$ of the HOCO and DOCO isomers are, thus, all $20-25 \mathrm{~cm}^{-1}$ below the corresponding $\operatorname{DVR}(6)$ results, ${ }^{51}$ obtained using their quartic force fields. The discrepancy between the VPT and VCI $\nu_{6}$ results was attributed ${ }^{38}$ to the transformed quartic force fields used in the VCI computations of Refs. 35,36. However, the torsional $\nu_{6}$ frequencies calculated for the quartic force fields expressed in terms of the bond-distance-bond-angle coordinates (used in the VPT calculations) are found to be smaller by only 3.2 and $3.7 \mathrm{~cm}^{-1}$ for respectively trans-HOCO and cisHOCO than the DVR(6) values obtained for the Morsecosine transformed quartic force fields (used in the VCI calculations). ${ }^{51}$ Note that the MULTIMODE computations in the single reference approach and reaction path approach yielded comparable results for $\nu_{6}$ in Ref. 38 .

The $\nu_{1}$ and $\nu_{2}$ fundamental transitions of the trans isomers agree within 12 and $10 \mathrm{~cm}^{-1}$, respectively, with the high resolution gas phase data. ${ }^{18-20}$ The experimental $\nu_{1}$ and $\nu_{2}$ frequencies available for the cis radicals isolated in solid $\mathrm{N}_{2}{ }^{50}$ agree with our values within 50 and $25 \mathrm{~cm}^{-1}$, respectively. In the $\mathrm{CO}$ matrix,${ }^{13}$ the bands were reported at 3316 [2456] and $1797[1798] \mathrm{cm}^{-1}$ for cis-HOCO [cis-DOCO], respectively. As seen, the interaction with $\mathrm{CO}$ substantially lowers the $\nu_{1}$ frequency. As a result of hydrogen bonding to the $\mathrm{CO}$ matrix through the $\mathrm{OH}$ group, large shifts $\Delta \nu_{1}$ occur relative to the free radical situation. This can be clearly seen on the example of trans-HOCO, for which the $\nu_{1}$ transition was observed at $3456,3571,3603$, and $3628 \mathrm{~cm}^{-1}$ in solid $\mathrm{CO},{ }^{13} \mathrm{~N}_{2},{ }^{50} \mathrm{Ar},{ }^{14}$ and $\mathrm{Ne},{ }^{49}$ yielding $\Delta \nu_{1}$ of $180,65,33$, and $8 \mathrm{~cm}^{-1}$, respectively. It is worth noting that the ratio $\nu_{1}\left(\mathrm{~N}_{2}\right) / \nu_{1}(\mathrm{CO})$, with the matrix medium shown in parentheses, calculated from the data of Refs. 13 and 50, equals to 1.03 for the isomers of both HOCO and DOCO. Using the ratio $\nu_{1}$ (gas) $/ \nu_{1}\left(\mathrm{~N}_{2}\right)=1.018$, valid for 
both trans-HOCO and trans-DOCO, we may estimate from $\nu_{1}$ in solid $\mathrm{N}_{2}$ the $\nu_{1}$ values of 3473 and $2568 \mathrm{~cm}^{-1}$ for free cis-HOCO and cis-DOCO, respectively. These are larger by 12 and $10 \mathrm{~cm}^{-1}$ than our $\nu_{1}$ results in Table III.

In accord with the large $\Delta \nu_{1}$ shifts in solid $\mathrm{CO}$, it is expected that all vibrations involving hydrogen are affected by the interaction with $\mathrm{CO}$, in particular the torsion. The torsional motion should appear more confined in the $\mathrm{CO}$ matrix than in the free radical, with the torsional fundamental at a larger frequency in the former case. The torsional $\nu_{6}$ transitions observed in the $\mathrm{CO}$ matrix ${ }^{13}$ at $472 \mathrm{~cm}^{-1}$ for trans-DOCO and at $497 \mathrm{~cm}^{-1}$ for cis-DOCO are noticeably larger, by 76 and $45 \mathrm{~cm}^{-1}$, than our respective theoretical values. In solid $\mathrm{Ne}^{49}$ and in solid $\mathrm{Ar},{ }^{14} \nu_{6}$ was reported only for trans-HOCO, at 508.1 and $515 \mathrm{~cm}^{-1}$, respectively, in close agreement with the theoretical results in Table III. Other experimental data for $\nu_{6}$ are not available.

\section{Low-frequency vibrations}

The low-lying in-plane vibrations $\nu_{3}, \nu_{4}$, and $\nu_{5}$ of $\mathrm{H} / \mathrm{DOCO}$ were characterized with the help of the zeroorder functions obtained in the adiabatic bend approximation for the planar arrangements $\left(\chi=0,180^{\circ}\right)$.

In the adiabatic bend approximation, the zero-order five-mode wavefunctions $\mid k ; \gamma)$ are described by the $k$ th state of the three-dimensional stretching vibration and the $\gamma$ th state of the two-dimensional bending vibration. ${ }^{47}$ The energy of the adiabatic state $\mid k ; \gamma)$ is $\varepsilon_{\text {adi }}$. The five-mode energies obtained including the stretch-bend coupling are designated with $\varepsilon^{(5 D)}$. In the $\operatorname{DVR}(6)$ approach, the full-dimensional vibrational energies $E^{(J, p)}$ are computed for $J=0$ and parity $p$ from $\varepsilon^{(5 D)}$ after the inclusion of the torsional contributions (the kinetic energy and kinetic coupling with the in-plane vibrations).

The energies $\varepsilon_{\text {adi }}, \varepsilon^{(5 D)}$, and $E^{(0,0)}$ are compared in Fig. 2 for $\nu_{3}, \nu_{4}$, and $\nu_{5}$. The adiabatic expansions in terms of $\mid k ; \gamma)$ for the five-mode states denoted by $a, b, c, d$ in Fig. 2 are summarized in Table IV. The latter two-component and three-component expansions provide more than $90 \%$ of the corresponding five-mode wavefunctions. The notation used by the code for $\mid k ; \gamma)$ is employed in Table IV. The adiabatic states $\mid 0 ; 1), \mid 0 ; 2)$, and $\mid 1 ; 0)$ arise from the $\theta_{2}, \theta_{1}$, and $R$ motion, respectively.

The coupling between the torsion and the $\nu_{3}, \nu_{4}, \nu_{5}$ in-plane vibrations is weak for $\mathrm{H} / \mathrm{DOCO}$, as evident by small differences (up to $3.8 \mathrm{~cm}^{-1}$ ) between the five-mode energies $\varepsilon^{(5 D)}$ and the accurate level energies $E^{(0,0)}$ in Fig. 2. On the other hand, nonadiabatic (stretch-bend mixing) effects appear very important. The levels $\varepsilon^{(5 D)}$ of HOCO correlated with $\nu_{4}$ and $\nu_{5}$ are pushed apart with respect to $\varepsilon_{\text {adi }}$ due to stretch-bend coupling. The difference $\Delta \varepsilon$ between $\varepsilon^{(5 D)}$ and $\varepsilon_{\text {adi }}$ amounts to -93 and $+47 \mathrm{~cm}^{-1}$ for trans-HOCO and to -92 and $+18 \mathrm{~cm}^{-1}$ for
cis-HOCO for $\nu_{5}$ and $\nu_{4}$, respectively. The corresponding zero-order eigenvector mixing in Table IV amounts to about $15 \%$. For $\nu_{4}$ of cis-HOCO, we also see the contribution from $\mid 0 ; 2)$ in the amount of $12 \%$. Here the $\mid 0 ; 2) / \mid 1 ; 0)\left(\nu_{4} / \nu_{3}\right)$ mixing acts in the opposite direction from $\mid 0 ; 1) / \mid 1 ; 0)\left(\nu_{4} / \nu_{5}\right)$, leading to a smaller $\Delta_{\varepsilon}$ value of $18 \mathrm{~cm}^{-1}$ compared to the value of $47 \mathrm{~cm}^{-1}$ for $\nu_{5}$.

For the DOCO isomers, the $\nu_{3} / \nu_{4}$ mixing becomes even more important. For $\nu_{3}$ and $\nu_{4}$, the differences $\Delta \varepsilon$ are found to be +83 and $-68 \mathrm{~cm}^{-1}$ for trans-DOCO and +94 and $-64 \mathrm{~cm}^{-1}$ for cis-DOCO. Inspection of Table IV also reveals for trans-DOCO nearly equal contributions from the adiabatic bending state $\mid 0 ; 2$ ) and the adiabatic stretching state $\mid 1 ; 0)$ in the five-mode state denoted by $b$, i.e. $\nu_{4}$. The strong resonance mixing in DOCO is due to mass effects: the frequencies $\left(\right.$ in $\mathrm{cm}^{-1}$ ) attributed to the one-dimensional $\left[R, \theta_{2}, \theta_{1}\right]$ motions are computed to be $[923,869,902]$ for trans-DOCO and [931, 895, 942] for cis-DOCO; to be compared with $[942,874,1204]$ for trans-HOCO and $[953,935,1186]$ for cis-HOCO.

To illustrate the importance of the $\nu_{3} / \nu_{4}$ mixing, the two-dimensional contour maps of the six-dimensional wavefunctions for the vibrational $\nu_{3}$ state of trans$\mathrm{HOCO}$, cis-HOCO, trans-DOCO, and cis-DOCO are shown in Fig. 3. Whereas $\mid 0 ; 2$ ) (angular character) provides the predominant contribution to $\nu_{3}$ of trans-HOCO, the stretching contribution from $\mid 1 ; 0)$ to $\nu_{3}$ increases from $12 \%$ for cis-HOCO to $45 \%$ for trans-DOCO to $58 \%$ for cis-DOCO (see Table IV). Having identified the origin of $\nu_{3}$ in trans-DOCO as $\left.\mid 0 ; 2\right)$ in Table IV, we may assume that the origin for $\nu_{4}$ of trans-DOCO is the zeroorder stretching state $\mid 1 ; 0)$.

It should be said that this type of analysis has led to the same conclusion regarding the properties of $\nu_{3}, \nu_{4}, \nu_{5}$ also in the case of the quartic force fields due to Fortenberry et al. ${ }^{35,36}$. For the latter PESs, the adiabatic expansions for the five-mode states considered in Fig. 2 were found to be almost identical to the results in Table IV for the PES developed here.

The nonadiabatic effects promote normal-mode character (collective nuclear motion) for the low-frequency $\nu_{3}, \nu_{4}, \nu_{5}$ vibrations involving both radial and bending degrees of freedom. Rotational excitation does not affect the intermode mixing in these states for both $\mathrm{HOCO}$ and DOCO, as seen from the corresponding adiabatic expansions calculated for $J=0-4$.

\section{Stretching $\nu_{1}$ and $\nu_{2}$ vibrations}

The high-frequency $\nu_{1}$ and $\nu_{2}$ stretching vibrations are found to be almost insensitive to the stretch-bend coupling. This is shown in Table $\mathrm{V}$, where we compare the stretching energies $\varepsilon_{s t r}^{(1 D)}$ from the one-dimensional calculations with the corresponding five-mode energies $\varepsilon^{(5 D)}$. As seen there, the two energy groups differ by at most $15 \mathrm{~cm}^{-1}$. Comparison of $\varepsilon^{(5 D)}$ with the accurate $\nu_{1}$ and 

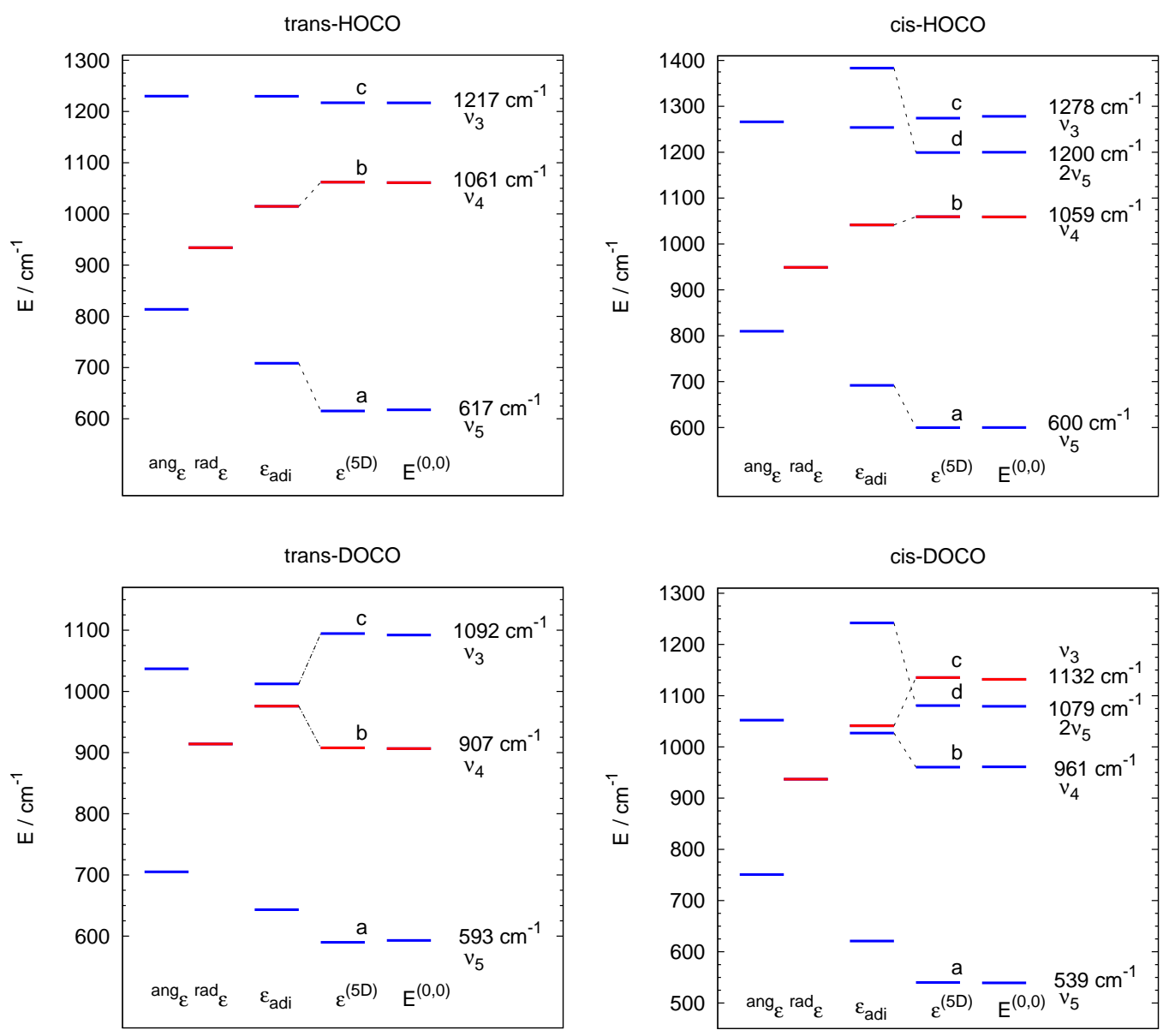

FIG. 2: Energies obtained in different stages of the $\operatorname{DVR}(6)$ calculation for the low-lying states of the in-plane vibrations of HOCO and DOCO. The energies calculated in the adiabatic bend approximation are denoted by $\varepsilon_{\text {adi }}$, whereas $\varepsilon^{(5 D)}$ and $E^{(0,0)}$ stand for the energies of the planar H/DOCO and the full-dimensional level energies, respectively. The energies ${ }^{\text {ang }} \varepsilon\left({ }^{\text {str }} \varepsilon\right)$ obtained using a single DVR point per radial (angular) degree of freedom are additionally shown. The adiabatic expansions for the five-mode states denoted here by $a, b, c, d$ are listed in Table IV.

TABLE IV: Adiabatic expansions in terms of the zero-order functions $\mid k ; \gamma)$ for the five-mode states $\varepsilon^{(5 D)}$ denoted by $a, b, c, d$ in Fig. 2. The zero-order function $\mid k ; \gamma)$ stands for a level with $k$ quanta in the 3D stretching vibration and $\gamma$ quanta in the $2 \mathrm{D}$ bending vibration. The zero-order contributions underlined here are all larger than $50 \%$. The five-mode states $a, b, c, d$ correlate with the full-dimensional $E^{(0,0)}$ vibrational $\nu_{5}, \nu_{4}, \nu_{3}, 2 \nu_{5}$ states, respectively.

\begin{tabular}{|c|c|c|c|c|c|}
\hline$\varepsilon^{(5 D)}$ & $E^{(0,0)}$ & trans-HOCO & cis-HOCO & trans-DOCO & cis-DOCO \\
\hline$a$ & $\nu_{5}$ & $\underline{0.90} \mid 0 ; 1)+0.40 \mid 1 ; 0)$ & $\underline{0.91} \mid 0 ; 1)+0.38 \mid 1 ; 0)$ & $\underline{0.93} \mid 0 ; 1)+0.33 \mid 1 ; 0)$ & $\underline{0.92} \mid 0 ; 1)+0.34 \mid 1 ; 0)$ \\
\hline$b$ & $\nu_{4}$ & $0.40 \mid 0 ; 1)-\underline{0.90} \mid 1 ; 0)$ & $\begin{array}{r}0.38 \mid 0 ; 1)-\underline{0.84} \mid 1 ; 0) \\
-0.35 \mid 0 ; 2)\end{array}$ & $\begin{array}{r}0.68 \mid 0 ; 2)-0.66 \mid 1 ; 0) \\
+0.30 \mid 0 ; 1)\end{array}$ & $\begin{array}{r}\underline{0.79 \mid 0 ; 2)-0.53 \mid 1 ; 0)} \\
+0.26 \mid 0 ; 1)\end{array}$ \\
\hline$c$ & $\nu_{3}$ & $\underline{0.96} \mid 0 ; 2)-0.07 \mid 1 ; 0)$ & $\begin{array}{r}\underline{0.91} \mid 0 ; 2)+0.35 \mid 1 ; 0) \\
-0.09 \mid 0 ; 1)\end{array}$ & $\begin{array}{r}\underline{0.72 \mid 0 ; 2)+0.67 \mid 1 ; 0)} \\
-0.16 \mid 0 ; 1)\end{array}$ & $\begin{array}{r}0.59 \mid 0 ; 2)+\underline{0.76} \mid 1 ; 0) \\
-0.22 \mid 0 ; 1)\end{array}$ \\
\hline$d$ & $2 \nu_{5}$ & & $\underline{0.82} \mid 0 ; 3)+0.48 \mid 1 ; 1)$ & & $\underline{0.85} \mid 0 ; 3)+0.43 \mid 1 ; 1)$ \\
\hline
\end{tabular}

$\nu_{2}$ energies given in Table III additionally indicates that the coupling with the torsional motion is weak.

The stretching $\nu_{1}$ and $\nu_{2}$ vibrations are of local-mode type, representing the $\mathrm{H}-\mathrm{O}$ and $\mathrm{C}=\mathrm{O}$ stretch, respec- tively. In agreement with this, the characterization of the states involving $\nu_{1}$ and $\nu_{2}$ was easily done with the help of the root mean square deviation $\sigma_{q}$, defined as $\sigma_{q}=\sqrt{\left\langle q^{2}\right\rangle-\langle q\rangle^{2}}$ for $q=d_{1}, d_{2}$. 

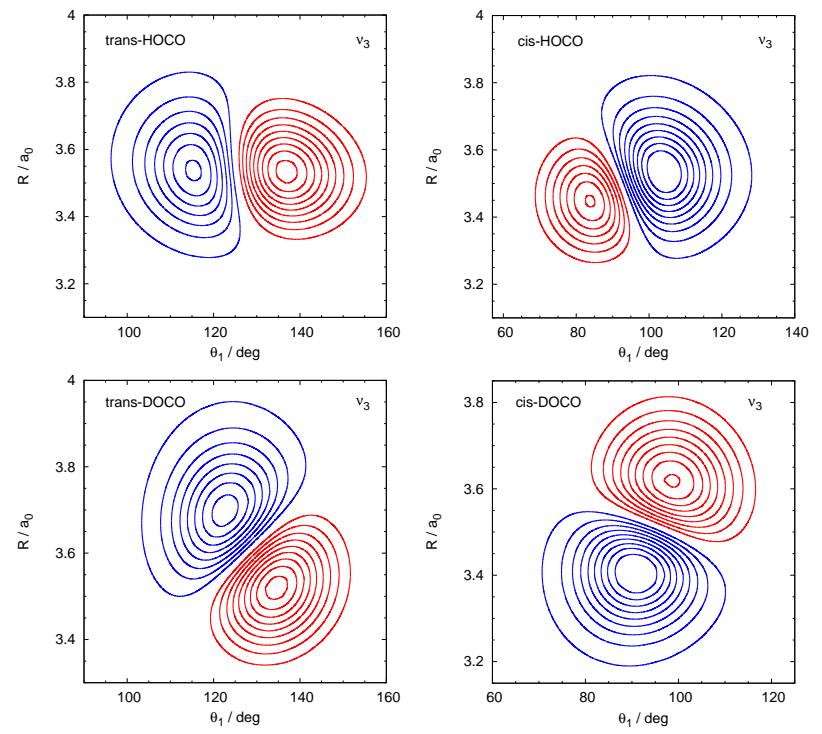

FIG. 3: Wavefunction contour maps for the vibrational $\nu_{3}$ state of trans-HOCO, cis-HOCO, trans-DOCO, and cisDOCO. The remaining four coordinates are kept constant at their equilibrium values. The contours seen in the $\left(\theta_{1}, R\right)$ plane show where the wavefunction takes from $-95 \%$ to $95 \%$ of its maximum value with the step of $10 \%$.

TABLE V: The fundamental high-frequency stretching transitions (in $\mathrm{cm}^{-1}$ ) from the one-dimensional $\varepsilon_{s t r}^{(1 D)}$ and fivedimensional $\varepsilon^{(5 D)}$ calculations, keeping the remaining coordinates constant at their equilibrium values.

\begin{tabular}{clllll}
\hline \hline & \multicolumn{2}{c}{ trans-HOCO } & & \multicolumn{2}{c}{ cis-HOCO } \\
\cline { 2 - 3 } \cline { 5 - 6 } & $\varepsilon_{\text {str }}^{(1 D)}$ & $\varepsilon^{(5 D)}$ & & $\varepsilon_{\text {str }}^{(1 D)}$ & $\varepsilon^{(5 D)}$ \\
\hline$\nu_{1}$ & 3667 & 3653 & & 3474 & 3459 \\
$\nu_{2}$ & 1867 & 1864 & & 1835 & 1824 \\
\hline & \multicolumn{2}{c}{ trans-DOCO } & & \multicolumn{2}{c}{ cis-DOCO } \\
\cline { 2 - 3 } & $\varepsilon_{\text {str }}^{(1 D)}$ & $\varepsilon^{(5 D)}$ & & $\varepsilon_{\text {str }}^{(1 D)}$ & $\varepsilon^{(5 D)}$ \\
\hline$\nu_{1}$ & 2702 & 2693 & & 2562 & 2556 \\
$\nu_{2}$ & 1867 & 1861 & & 1835 & 1829 \\
\hline \hline
\end{tabular}

The wavefunctions corresponding to the $\nu_{1}$ and $\nu_{2}$ fundamentals of HOCO possess a large dominant contribution (larger than $95 \%$ ). The same was also found for the quartic force fields of Fortenberry and others. ${ }^{35,36}$ Note that $\nu_{1}$ of cis-HOCO was identified as a mixed state in the calculations of Wang and others. ${ }^{38}$

\section{B. Torsion}

The torsional motion of HOCO is described by an anharmonic double-minimum torsional potential with a non-planar barrier between the trans and cis arrangements. The expectation value $\langle\chi\rangle$ of the torsional angle is found to be about $165^{\circ}$ and $10^{\circ}$ in the vibrational ground state of the trans and cis isomers, respectively. It amounts to about $155^{\circ}$ and $20^{\circ}$ in the first excited torsional states.

HOCO levels residing either in the trans or the cis well were found by inspection of the expectation value $\langle\chi\rangle$ of the torsional angle. The extent of delocalization of the $n$th state is quanified with the help of the integrated wavefunction probability amplitude $P_{n}^{(a, b)}$,

$$
P_{n}^{(a, b)}=\int_{a}^{b} d \chi \int\left|\psi_{n}\right|^{2} d \Omega
$$

giving the localization probability for the $\chi$ interval $(a, b)$. In Eq. (4), $\Omega$ stands for all other coordinates. The localization probability in the trans well is obtained setting $a=\chi_{s p}$ and $b=180^{\circ}$, where $\chi_{s p}$ gives the position of the torsional saddle point (see Table II). In the diatomdiatom description, $\chi_{s p}=79.1^{\circ}$.

The vibrational ground state of trans-HOCO and the vibrational ground state of cis-HOCO lie at $4562 \mathrm{~cm}^{-1}$ and $5100 \mathrm{~cm}^{-1}$, respectively. Although both ground states are placed above the electronic torsional saddle point at $3270 \mathrm{~cm}^{-1}$ (Table II), we found that the majority of the calculated HOCO levels are localized in one of the two wells even at high vibrational energy. This high-energy state localization can be easily understood in terms of effective (adiabatic) torsional potentials ${ }^{a d i} V^{i}={ }^{a d i} V^{i}(\chi)$. The profiles ${ }^{a d i} V^{i}$ differ from the torsional minimum energy path in the energy of the $i$ th state of the in-plane five-mode vibration. The barrier height ${ }^{a d i} E_{\text {har }}^{i}$ measured with respect to the trans minimum of ${ }^{a d i} V^{i}$ may also differ from the corresponding MEP value. For instance, along the effective groundstate torsional profile ${ }^{a d i} V^{0}$, the cis-trans separation and ${ }^{a d i} E_{b a r}^{0}$ amount respectively to $520[548] \mathrm{cm}^{-1}$ and 3109 [3135] $\mathrm{cm}^{-1}$ for HOCO [DOCO], being thus by 83 [55] $\mathrm{cm}^{-1}$ and $161[135] \mathrm{cm}^{-1}$ smaller than the corresponding electronic-potential values of $603 \mathrm{~cm}^{-1}$ and $3270 \mathrm{~cm}^{-1}$ in Table II.

The torsional saddle point on the effective ground-state profile ${ }^{a d i} V^{0}$ for HOCO is at $7410 \mathrm{~cm}^{-1}$. Below this energy, there are 45 even-parity and 27 odd-parity eigenstates. Among these, 45 states are identified as strictly trans-HOCO states $\left[P_{n}^{\left(\chi_{s p}, 180^{\circ}\right)}=1\right]$ and 24 as strictly cis-HOCO states $\left[P_{n}^{\left(\chi_{s p}, 180^{\circ}\right)}=0\right]$. The remaining 3 states have a non-zero wavefunction probability amplitude in both well regions. The first tunneling cis state $n^{(0,0)}=35$ at $7174 \mathrm{~cm}^{-1}$ and the first tunneling trans state $n^{(0,0)}=36$ at $7176 \mathrm{~cm}^{-1}$ have $P_{n}^{\left(\chi_{s p}, 180^{\circ}\right)}$ of 0.33 and 0.64 , respectively. The odd-parity level $n^{0,1}=26$ of HOCO at $7368 \mathrm{~cm}^{-1}$ has $P_{n}^{\left(\chi_{s p}, 180^{\circ}\right)}$ of 0.24 . DOCO has 69 even-parity and 45 odd-parity states below the corresponding torsional saddle point at $6773 \mathrm{~cm}^{-1}$ on ${ }^{a d i} V^{0}$, out of which 74 states belong strictly to transDOCO and 36 strictly to cis-DOCO. The remaining 4 


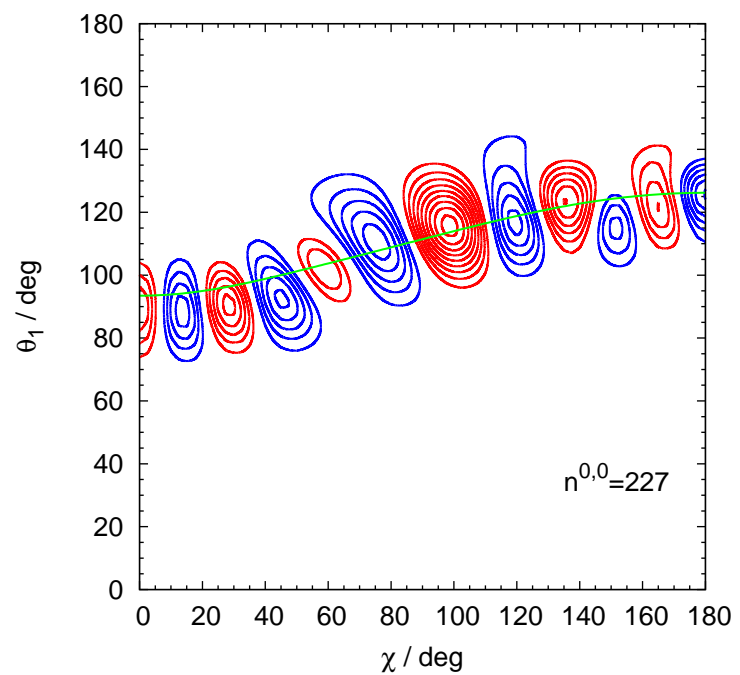

FIG. 4: Two-dimensional contour map of the full-dimensional wavefunction for the torsional even-parity state $11 \nu_{6}$ of $\mathrm{HOCO}$, obtained for the remaining four coordinates kept constant at their equilibrium values. The contours indicate where the wavefunction assumes from $-95 \%$ to $95 \%$ of its maximum value with the step of $10 \%$. The solid (green online) line shows the variation of the angle $\theta_{1}$ along the torsional minimum energy path.

TABLE VI: Effective torsional frequencies (in $\mathrm{cm}^{-1}$ ) calculated according to Eq. (5).

\begin{tabular}{lcccc}
\hline \hline & trans-HOCO & cis-HOCO & trans-DOCO & cis-DOCO \\
\hline$\nu_{1}$ & 498 & 548 & 394 & 456 \\
$\nu_{2}$ & 505 & 542 & 396 & 451 \\
$\nu_{3}$ & 504 & 543 & 391 & 444 \\
$\nu_{4}$ & 503 & 530 & 393 & 448 \\
$\nu_{5}$ & 511 & 543 & 402 & 449 \\
\hline \hline
\end{tabular}

are tunneling states: two even-parity states at 6544 and $6581 \mathrm{~cm}^{-1}$ with $P_{n}^{\left(\chi_{s p}, 180^{\circ}\right)}$ of respectively 0.88 and 0.12 and two odd-parity states at 6719 and $6737 \mathrm{~cm}^{-1}$ with $P_{n}^{\left(\chi_{s p}, 180^{\circ}\right)}$ of respectively 0.28 and 0.90 .

The tunneling states of HOCO and DOCO described in the previous paragraph are found to be pure torsional levels $\left(\nu_{i}=0\right)$. They lie in the vicinity of the ground state adiabatic barrier. Pure torsional states above ${ }^{a d i} V^{0}(\chi=$ $\left.\chi_{s p}\right)$ are all delocalized over both minima. To illustrate this, we show in Fig. 4 the wavefunction contour map for the even-parity HOCO torsional state $11 \nu_{6}$ lying at 9228 $\mathrm{cm}^{-1}$.

The effective torsional frequency $\nu_{6}^{e f f}\left(\nu_{i}\right)$ upon the excitation of the in-plane mode $\nu_{i}$, estimated as the energy difference between the odd-parity and even-parity values,

$$
\nu_{6}^{e f f}\left(\nu_{i}\right)=E_{\nu_{i}+\nu_{6}}^{(0,1)}-E_{\nu_{i}}^{(0,0)}
$$

are summarized in Table VI. There we see that the largest deviation of $13 \mathrm{~cm}^{-1}$ is between the fundamental torsional frequency from Table III and $\nu_{6}^{\text {eff }}\left(\nu_{i}\right)$ for $\nu_{i}=\nu_{4}$ of cis-HOCO. This is caused by the zero-order $\nu_{4} / 2 \nu_{6}$ mixing, as evident from the following adiabatic expansions

$$
\begin{aligned}
\left|\nu_{4}\right\rangle= & \underline{0.85} \mid 2,0 ; 0)+0.50 \mid 0,1 ; 0) \\
& +0.11 \mid 4,0 ; 0) \\
\left|2 \nu_{6}\right\rangle= & -0.52 \mid 2,0 ; 0)+\underline{0.84} \mid 0,1 ; 0) \\
& +0.15 \mid 4,0 ; 0)
\end{aligned}
$$

whereas we have

$$
\left.\left.\left|\nu_{3}\right\rangle=\underline{0.98} \mid 4,0 ; 0\right)-0.18 \mid 0,1 ; 0\right) .
$$

The zero-order functions $\mid i, \alpha ; p)$, computed in the adiabatic torsion approximation, describe the adiabatic levels with $i$ quanta in the five-mode in-plane vibration and $\alpha$ quanta in the torsion for a given parity $p$. In Eq. (6), $\left.\left.\left.\mid 0,1 ; 0)=\mid 2 \nu_{6}\right), \mid 2,0 ; 0\right)=\mid \nu_{4}\right)$, and $\left.\left.\mid 4,0 ; 0\right)=\mid \nu_{3}\right)$ holds for even parity $(p=0)$. The levels $2 \nu_{6}, \nu_{4}$, and $\nu_{3}$ are at 1041,1059 , and $1278 \mathrm{~cm}^{-1}$, respectively. For the quartic force field of Fortenberry et $a l^{36}$ for cis-HOCO, the $2 \nu_{6}$ level was found to exhibit only $\nu_{3} / 2 \nu_{6}$ mixing (to an extent of $5 \%$ ) and no $\nu_{4} / 2 \nu_{6}$ mixing. ${ }^{51}$ The latter finding stems from the fact that the quartic force field of Fortenberry et al. supports a torsional frequency that is $21 \mathrm{~cm}^{-1}$ larger than $\nu_{6}$ for the PES developed here. The $\nu_{4}, 2 \nu_{6}, \nu_{3}$ levels of cis-HOCO and the PES of Fortenberry et al. are calculated to be 1046, 1099, and 1282 $\mathrm{cm}^{-1}$, respectively. ${ }^{51}$

For the present RCCSD $(\mathrm{T}) / \mathrm{cc}-\mathrm{pVQZ} \mathrm{PES}$, the $\nu_{4} / 2 \nu_{6}$ mixing in cis-DOCO was found to be less pronounced (to an extent of $6 \%$ ). On the other hand, $\nu_{2} / \nu_{3}+2 \nu_{6}$ for trans-DOCO and $\nu_{2} / \nu_{4}+2 \nu_{6}$ for cis-DOCO appear strong with a mixing of $35 \%$ and $24 \%$, respectively.

\section{Rotational constants}

The rotational constants for the vibrational ground state of the trans and cis isomers of HOCO and DOCO are summarized in Table VII. Our values are obtained from $J=0,1$ calculations with the $\mathrm{DVR}(+\mathrm{R})+\mathrm{FBR}$ approach. The theoretical results of Fortenberry et al. ${ }^{35,36}$ were derived by means of vibrational second order perturbation theory.

The rotational constants $A$ from the present work agree within about $30,600,100$, and $300 \mathrm{MHz}$ with the experimental finding ${ }^{16,17,21}$ for trans-HOCO, cis-HOCO, transDOCO, and cis-DOCO, respectively. Our rotational constants $B$ and $C$ are approximately $50 \mathrm{MHz}$ smaller than the experimental counterpart. With respect to the experiment, the rotational constants $A$ due to Fortenberry et al. are larger by about $500 \mathrm{MHz}$ and $200 \mathrm{MHz}$ for respectively trans-HOCO and cis-HOCO, whereas the agreement is within $20 \mathrm{MHz}$ for $B$ and $C$. 
TABLE VII: Rotational constants (in $\mathrm{cm}^{-1}$ ) for the vibrational ground state of trans-HOCO, cis-HOCO, trans-DOCO, and cis-DOCO. The values in parentheses are given in $\mathrm{MHz}$.

\begin{tabular}{|c|c|c|c|c|c|c|c|c|c|c|c|c|}
\hline & \multicolumn{3}{|c|}{ trans-HOCO } & \multicolumn{3}{|c|}{ cis-HOCO } & \multicolumn{3}{|c|}{ trans-DOCO } & \multicolumn{3}{|c|}{ cis-DOCO } \\
\hline & $A$ & $B$ & $C$ & $A$ & $B$ & $C$ & $A$ & $B$ & C & $A$ & $B$ & $C$ \\
\hline \multicolumn{13}{|c|}{ theoretical results } \\
\hline This work & $\begin{array}{r}5.5972 \\
(167800)\end{array}$ & $\begin{array}{r}0.3797 \\
(11383)\end{array}$ & $\begin{array}{r}0.3550 \\
(10643)\end{array}$ & $\begin{array}{r}4.7476 \\
(142328)\end{array}$ & $\begin{array}{r}0.3898 \\
(11687)\end{array}$ & $\begin{array}{r}0.3596 \\
(10782)\end{array}$ & $\begin{array}{r}5.1555 \\
(154557)\end{array}$ & $\begin{array}{r}0.3550 \\
(10642)\end{array}$ & $\begin{array}{l}0.3316 \\
(9943)\end{array}$ & $\begin{array}{r}3.6617 \\
(109775)\end{array}$ & $\begin{array}{r}0.3794 \\
(11374)\end{array}$ & $\begin{array}{r}0.3432 \\
(10289)\end{array}$ \\
\hline Refs. 35,36 & $\begin{array}{r}5.6128 \\
(168266) \\
\end{array}$ & $\begin{array}{r}0.3819 \\
(11448) \\
\end{array}$ & $\begin{array}{r}0.3570 \\
(10702) \\
\end{array}$ & $\begin{array}{r}4.7750 \\
(143152) \\
\end{array}$ & $\begin{array}{r}0.3922 \\
(11757) \\
\end{array}$ & $\begin{array}{r}0.3618 \\
(10846) \\
\end{array}$ & & & & & & \\
\hline \multicolumn{13}{|c|}{ experimental results } \\
\hline Ref. 21 & $\begin{array}{r}5.5961 \\
(167768)\end{array}$ & $\begin{array}{r}0.3814 \\
(11433)\end{array}$ & $\begin{array}{r}0.3565 \\
(10687)\end{array}$ & $\begin{array}{r}4.7681 \\
(142945)\end{array}$ & $\begin{array}{r}0.3916 \\
(11739)\end{array}$ & $\begin{array}{r}0.3612 \\
(10830)\end{array}$ & $\begin{array}{r}5.1597 \\
(154686)\end{array}$ & $\begin{array}{r}0.3564 \\
(10686)\end{array}$ & $\begin{array}{l}0.3329 \\
(9982)\end{array}$ & $\begin{array}{r}3.6726 \\
(110100)\end{array}$ & $\begin{array}{r}0.3810 \\
(11423)\end{array}$ & $\begin{array}{r}0.3446 \\
(10332)\end{array}$ \\
\hline Refs. 16,17 & $\begin{array}{r}5.5961 \\
(167766)\end{array}$ & $\begin{array}{r}0.3814 \\
(11433)\end{array}$ & $\begin{array}{r}0.3565 \\
(10687)\end{array}$ & & & & $\begin{array}{r}5.1598 \\
(154686)\end{array}$ & $\begin{array}{r}0.3564 \\
(10686)\end{array}$ & $\begin{array}{l}0.3329 \\
(9982)\end{array}$ & & & \\
\hline
\end{tabular}

TABLE VIII: Vibrationally averaged internal geometries expressed in terms of the bond-distance-bond-angle coordinates $r_{1}, r_{2}, r_{3}, \alpha, \beta$ for the vibrational ground state of trans-HOCO, cis-HOCO, trans-DOCO, and cis-DOCO. The angle $\chi$ is the dihedral angle in the orthogonal diatom-diatom description. The results obtained for the present RCCSD(T) PES and for the previous quartic force fields ${ }^{35,36}$ are shown in the columns denoted by I and II, respectively. The angles are given in degree and the distances in $\mathrm{a}_{0}$.

\begin{tabular}{|c|c|c|c|c|c|c|c|c|}
\hline & \multicolumn{2}{|c|}{ trans-HOCO } & \multicolumn{2}{|c|}{ cis-HOCO } & \multicolumn{2}{|c|}{ trans-DOCO } & \multicolumn{2}{|c|}{ cis-DOCO } \\
\hline & I & II & I & II & I & II & I & II \\
\hline$\langle\chi\rangle$ & 165.0 & 164.8 & 12.3 & 12.1 & 166.5 & 166.4 & 10.7 & 10.6 \\
\hline$\langle\alpha\rangle$ & 107.4 & 107.7 & 107.8 & 108.1 & 107.4 & 107.7 & 107.7 & 108.0 \\
\hline$\langle\beta\rangle$ & 126.8 & 126.7 & 130.1 & 130.1 & 126.8 & 126.7 & 130.0 & 130.0 \\
\hline$\left\langle r_{1}\right\rangle$ & 1.854 & 1.853 & 1.875 & 1.873 & 1.845 & 1.843 & 1.864 & 1.862 \\
\hline$\left\langle r_{2}\right\rangle$ & 2.558 & 2.552 & 2.533 & 2.523 & 2.558 & 2.552 & 2.533 & 2.523 \\
\hline$\left\langle r_{3}\right\rangle$ & 2.235 & 2.229 & 2.245 & 2.239 & 2.235 & 2.229 & 2.245 & 2.239 \\
\hline
\end{tabular}

For both forms of HOCO and DOCO, we give in Table VIII the vibrationally averaged geometries for the vibrational ground state obtained for the present $\operatorname{RCCSD}(\mathrm{T}) / \mathrm{cc}-\mathrm{pVQZ}$ PES and the previous quartic force fields. ${ }^{35,36}$ The expectation values of the bond-distancebond-angle coordinates were obtained by their direct averaging, i.e. they were not evaluated from the expectation values of the orthogonal coordinates employed in the rovibrational calculations. The differences between the expectation geometries for the two PESs are comparable with the differences seen between the corresponding equilibrium structures in Table II. Comparison of Tables II and VIII shows an increase of $\left\langle r_{1}\right\rangle,\left\langle r_{2}\right\rangle$, and $\left\langle r_{3}\right\rangle$ with respect to $r_{1}^{e}, r_{2}^{e}, r_{3}^{e}$ by about $0.035,0.02$, and $0.01 \mathrm{a}_{0}$, respectively. The angles $\langle\alpha\rangle$ and $\langle\beta\rangle$ are decreased with respect to $\alpha^{e}$ and $\beta^{e}$ by $0.3^{\circ}$ or less.

Oyama et al. ${ }^{21}$ derived a $r_{0}$ structure given by $(1.841$ $\mathrm{a}_{0}, 2.536 \mathrm{a}_{0}, 2.232 \mathrm{a}_{0}, 107.4^{\circ}, 127.4^{\circ}$ ) for trans-HOCO, where the internal geometry $\left(r_{1}, r_{2}, r_{3}, \alpha, \beta\right)$ is given in terms of the bond-distance-bond-angle coordinates. For cis-HOCO, they found $\left(1.873 \mathrm{a}_{0}, 2.511 \mathrm{a}_{0}, 2.237 \mathrm{a}_{0}\right.$, $\left.107.3^{\circ}, 131.1^{\circ}\right)$. Both conformers were assumed to have a planar structure. These $r_{0}$ structures were derived also assuming the theoretical UCCSD(T)-F12/aug-cc-pVTZ values for $r_{2}$, which is thus more comparable with the value for $r_{2}^{e}$ in TableII. Regarding the non-adjusted parameters in the $r_{0}$ structures, we note a difference of $1^{\circ}$ for $\beta=\angle(\mathrm{O}-\mathrm{C}-\mathrm{O})$ with respect to the results of Table VIII. Upon deuteration, only $\left\langle r_{1}\right\rangle$ in Table VIII is changed by an amount of $0.01 \mathrm{a}_{0}$, as assumed in Ref. 21 . The contributions of the zero-point vibrations were not considered in Ref. 21.

The experimental rotational constants available for HOCO and DOCO do not provide all the information needed to derive complete experimental structures of $\mathrm{cis}$ and trans conformers since $I_{1}=I_{2}+I_{3}$ holds for the principal moments of inertia $I_{1}, I_{2}, I_{3}$ of planar tetratomic molecules. In other words, (at least) one of the internal coordinates has to be assumed known in a fitting procedure. After several tests, the distance $r_{3}$ was chosen to be kept constant: we used $r_{3}=2.2236 \mathrm{a}_{0}$ for trans-HOCO and $r_{3}=2.2335 \mathrm{a}_{0}$ for cis-HOCO as the mean of $r_{3}^{e}$ from our work and Refs. 35,36 from Table II. Combining the theoretical ground-state vibrational corrections $A_{e}-A_{0}, B_{e}-B_{0}, C_{e}-C_{0}$ obtained for the RCCSD(T)/cc-pVQZ PES with the experimental ground-state rotational constants due to Oyama et $a l^{21}$ we finally derived the semi-experimental equilibrium $r_{e}$ structure $\left(r_{1}^{e}, r_{2}^{e}, \alpha^{e}, \beta^{e}\right)$ given by $\left(1.817 \mathrm{a}_{0}, 2.534 \mathrm{a}_{0}\right.$, $\left.107.9^{\circ}, 126.9^{\circ}\right)$ for trans-HOCO and by $\left(1.838 \mathrm{a}_{0}, 2.505\right.$ $\left.\mathrm{a}_{0}, 108.2^{\circ}, 130.4^{\circ}\right)$ for cis-HOCO. The latter structures 
TABLE IX: Geometric parameters of selected stationary points calculated at the RCCSD(T)/cc-pVQZ level of theory. The energy measured relative to the energy of the trans minimum is denoted by $\mathrm{E}_{r e l}$.

\begin{tabular}{lrrrrrrr}
\hline \hline & $r_{1} / a_{0}$ & $r_{2} / a_{0}$ & $r_{3} / a_{0}$ & $\alpha / \operatorname{deg}$ & $\beta / \operatorname{deg}$ & $\tau / \operatorname{deg}$ & $\mathrm{E}_{\text {rel }} / \mathrm{cm}^{-1}$ \\
\hline $\mathrm{H}+\mathrm{CO}_{2}$ & 2.1971 & 2.1971 & & 180.0 & & 2150 \\
$\mathrm{HCO}_{2}$ & 2.6696 & 2.3125 & 2.3125 & 51.7 & 145.1 & 180 & 5825 \\
$\mathrm{HO}+\mathrm{CO}$ & 1.8322 & & 2.1381 & & & & 9868 \\
\hline \hline
\end{tabular}

are in very good agreement (within $0.009 \mathrm{a}_{0}$ and $0.3^{\circ}$ ) with the results of Table II. Analytical expressions for the principal moments of inertia in the bond-distance-bondangle description and their numerical partial derivatives with respect to the geometric parameters in combination with a Levenberg-Marquardt nonlinear least-squares algorithm ${ }^{44}$ were used to compute the semi-experimental equilibrium geometry.

\section{FINAL REMARKS}

The RCCSD(T)/cc-pVQZ potential energy surface developed for the HOCO radical was combined with advanced strategies for the calculation of rotation-vibration levels of tetratomic molecules. Our study showed that the main features of the energy spectum of $\mathrm{H} / \mathrm{DOCO}$ are large amplitude torsional motion and anharmonic mixing of the low-frequency stretch with the bending modes, whereas the coupling between the torsion and the lowfrequency in-plane modes appears to be less pronounced in the region of the fundamental transitions. For a given state of the in-plane vibration, the torsional structure is found to exhibit two limiting cases associated with oscillator and rotor spectral patterns.

Several additional studies on the HOCO system are in progress. The first part is concerned with the improvement of the functional form used here to represent the potential energy surface. The analytical expansion of Eq. (2) is invariant under the spatial inversion $(\tau \rightarrow 2 \pi-\tau)$. However, it is not independent from the torsional angle for linear arrangements $(\alpha, \beta=0, \pi)$. These two properties of $V$, which are general features of any tetratomic molecule, ${ }^{52}$ have to be combined together to obtain a physically justified potential-energy representation. Molecular arrangements with a strictly linear HOC $(\alpha=\pi)$ or $\mathrm{OHC}(\alpha=0)$ skeleton, important in the direction of the $\mathrm{HO}+\mathrm{CO}$ channel over the configuration space of the weakly bound complexes $\mathrm{HO}-\mathrm{CO}$ and $\mathrm{OH}-\mathrm{CO},{ }^{6}$ occur at roughly $10000 \mathrm{~cm}^{-1}$. The exit channel $\mathrm{HO}+\mathrm{CO}$ is also seen at this energy in Table IX. This provides an explanation why only $a b$ initio points with energies up to $10000 \mathrm{~cm}^{-1}$ above the trans minimum were considered in the current fit. The further development of the PES is based on almost twice as many energy points, which have been already computed.

$A b$ initio points along the torsional minimum energy path were explicitly included in the fitting procedure. The number of non-planar arrangements was somewhat larger than 2000. Considering that the torsional motion above the torsional saddle point experiences a constant potential-energy contribution in the free-rotor region, we may state that our PES provides a reliable description useful for studying at least general torsional properties. In that respect, the $\mathrm{HOCO}$ system nicely complements the other class of tetratomic molecules, provided by the CHNO family. ${ }^{53}$ In Table IX, we also list the optimum structure of the local minimum $\mathrm{HCO}_{2}$, which can be related to the trans and cis forms by hydrogen migration. This is another challenge for improvements of the potential energy representation, since the two (different) oxygen atoms exposed to different interactions in $\mathrm{HOCO}$ become equivalent in $\mathrm{HCO}_{2}$.

The rovibrational states of HOCO pose also a challenge for numerically exact methods. The reason for this is due to the large-amplitude torsional motion, exhibiting the onset of a rotor-like energy structure below relatively high barriers to linearity. To treat this, we need primary functions capable to capture both the one-dimensional torsional character and the rotational character of the two-dimensional vibrational angular momentum. From a practical point of view, basis sets comprising a large number of functions are required to handle the rovibrational motion of $\mathrm{HOCO}$ in a numerically exact fashion. We completed the rovibrational calculations for up to $J=4$ in both parity. The results of this work, under analysis now, will be discussed in more detail elsewhere.

A final comment concerns the asymptote $\mathrm{H}+\mathrm{CO}_{2}$, seen at $2150 \mathrm{~cm}^{-1}$ above the trans minimum in Table IX. The ground-state energy of $\mathrm{CO}_{2}$ is calculated to be $2536 \mathrm{~cm}^{-1}$ employing the $\mathrm{CO}_{2}$ potential energy surface of Carter and Murrell ${ }^{54}$ and our DVR-DGB approach for triatomic molecules. ${ }^{55,56}$ In view of this estimate, states localized in the HOCO region at energies above $4686 \mathrm{~cm}^{-1}$ are, rigorously speaking, resonance states embedded into the continuum. For the RCCSD $(\mathrm{T}) / \mathrm{cc}-\mathrm{pVQZ}$ PES, only the ground state of trans-HOCO at $4562 \mathrm{~cm}^{-1}$ appears as a true bound state.

\section{Acknowledgments}

The author is grateful to Professor Peter Botschwina for very helpful suggestions and stimulating interest in this work. 
* Corresponding author; Electronic address: mladenov@ univ-mlv.fr

1 Smith, I. W. M.; Zellner, R. Rate Measurements of Reactions of $\mathrm{OH}$ by Resonance Absorption. Part 2.-Reactions of $\mathrm{OH}$ with $\mathrm{CO}, \mathrm{C}_{2} \mathrm{H}_{4}$ and $\mathrm{C}_{2} \mathrm{H}_{2}$, J. Chem. Soc., Faraday Trans. 2, 1973, 69, 1617-1627.

2 Smith, I. W. The Mechanism of the $\mathrm{OH}+\mathrm{CO}$ Reaction and the Stability of the HOCO Radical, Chem. Phys. Lett. 1977, 49(1), 112-115.

3 Ruscic, B.; Schwarz, M.; Berkowitz, J. A Photoionization Study of the COOH Species, J. Chem. Phys. 1989, 91(11), 6780-6785.

4 Scherer, N. F.; Sipes, C.; Bernstein, R. B.; Zewail, A. H. Real-Time Clocking of Bimolecular Reactions: Application to $\mathrm{H}+\mathrm{CO}_{2}$, J. Chem. Phys. 1990, 92(9), 5239-5259.

${ }^{5}$ Petty, J. T.; Harrison, J. A.; Moore, C. B. Reactions of Trans-Hydroxycarbonyl Radical Studied by Infrared Spectroscopy, J. Phys. Chem. 1993, 97(43), 11194-11198.

${ }^{6}$ Lester, M. I.; Pond, B. V.; Anderson, D. T.; Harding, L. B.; Wagner, A. F. Exploring the $\mathrm{OH}+\mathrm{CO}$ Reaction Coordinate via Infrared Spectroscopy of the $\mathrm{OH}-\mathrm{CO}$ Reactant Complex, J. Chem. Phys. 2000, 113(22), 9889-9892.

7 Miller, J. A.; Kee, R. J.; Westbrook, C. K. Chemical Kinetics and Combustion Modeling, Annu. Rev. Phys. Chem. 1990, 41(1), 345-387.

8 Frost, M. J.; Sharkey, P.; Smith, I. W. M. Energy and Structure of the Transition States in the Reaction $\mathrm{OH}+$ $\mathrm{CO} \rightarrow \mathrm{H}+\mathrm{CO}_{2}$, Faraday Discuss. Chem. Soc. 1991, 91, 305-317.

9 Ravishankara, A.; Thompson, R. Kinetic Study of the Reaction of $\mathrm{OH}$ with $\mathrm{CO}$ from 250 to $1040 \mathrm{~K}$, Chem. Phys. Lett. 1983, 99(5-6), 377-381.

10 Fulle, D.; Hamann, H. F.; Hippler, H.; Troe, J. High Pressure Range of Addition Reactions of HO. II. Temperature and Pressure Dependence of the Reaction $\mathrm{HO}+\mathrm{CO}$ $\Rightarrow \mathrm{HOCO} \rightarrow \mathrm{H}+\mathrm{CO}_{2}$, J. Chem. Phys. 1996, 105(3), 9831000 .

11 Golden, D. M.; Smith, G. P.; McEwen, A. B.; Yu, C.L.; Eiteneer, B.; Frenklach, M.; Vaghjiani, G. L.; Ravishankara, A. R.; Tully, F. P. OH(OD) + CO: Measurements and an Optimized RRKM Fit, J. Phys. Chem. A 1998, 102(44), 8598-8606.

12 Guo, H. Quantum Dynamics of Complex-Forming Bimolecular Reactions, Int. Rev. Phys. Chem. 2012, 31(1), $1-68$.

13 Milligan, D. E.; Jacox, M. E. Infrared Spectrum and Structure of Intermediates in the Reaction of $\mathrm{OH}$ with $\mathrm{CO}$, J. Chem. Phys. 1971, 54(3), 927-942.

14 Jacox, M. E. The Vibrational Spectrum of the t-HOCO Free Radical Trapped in Solid Argon, J. Chem. Phys. 1988, 88(8), 4598-4607.

15 Radford, H. E.; Wei, W.; Sears, T. J. The Rotational Spectrum of trans-HOCO and DOCO, J. Chem. Phys. 1992, 97(6), 3989-3995.

16 Sears, T. J.; Radford, H. E.; Moore, M. A. b-Dipole Transitions in trans-HOCO Observed by Far Infrared Laser Magnetic Resonance, J. Chem. Phys. 1993, 98(9), 6624-6631.

17 Radford, H.; Moore, M.; Sears, T.; Grussdorf, J.; Nolte, J.; Temps, F. Far-Infrared Laser Magnetic Resonance of X 2A' trans-DOCO, J. Mol. Spectrosc. 1994, 165(1), 137-149.

18 Sears, T. J.; Fawzy, W. M.; Johnson, P. M. Transient Diode
Laser Absorption Spectroscopy of the $\nu_{2}$ Fundamental of trans-HOCO and DOCO, J. Chem. Phys. 1992, 97(6), 3996-4007.

19 Petty, J.; Moore, C. Transient Infrared Absorption Spectrum of the $\nu_{1}$ Fundamental of trans-HOCO, J. Mol. Spectrosc. 1993, 161(1), 149-156.

20 Petty, J. T.; Moore, C. B. Transient Infrared Absorption Spectrum of the $\nu_{1}$ Fundamental of trans-DOCO, J. Chem. Phys. 1993, 99(1), 47-55.

21 Oyama, T.; Funato, W.; Sumiyoshi, Y.; Endo, Y. Observation of the Pure Rotational Spectra of trans- and cisHOCO, J. Chem. Phys. 2011, 134(17), 174303.

22 Johnson, C. J.; Harding, M. E.; Poad, B. L. J.; Stanton, J. F.; Continetti, R. E. Electron Affinities, Well Depths, and Vibrational Spectroscopy of cis- and trans-HOCO, J. Am. Chem. Soc. 2011, 133(49), 19606-19609.

23 Schatz, G. C.; Fitzcharles, M. S.; Harding, L. B. Stateto-State Chemistry with Fast Hydrogen Atoms. Reaction and Collisional Excitation in $\mathrm{H}+\mathrm{CO}_{2}$, Faraday Discuss. Chem. Soc. 1987, 84, 359-369.

24 Yu, H.-G.; Muckerman, J. T.; Sears, T. J. A Theoretical Study of the Potential Energy Surface for the Reaction $\mathrm{OH}+\mathrm{CO} \rightarrow \mathrm{H}+\mathrm{CO}_{2}$, Chem. Phys. Lett. 2001, 349(5-6), 547-554.

${ }^{25}$ Lakin, M. J.; Troya, D.; Schatz, G. C.; Harding, L. B. A Quasiclassical Trajectory Study of the Reaction $\mathrm{OH}+\mathrm{CO}$ $\rightarrow \mathrm{H}+\mathrm{CO}_{2}$, J. Chem. Phys. 2003, 119(12), 5848-5859.

26 Valero, R.; van Hemert, M. C.; Kroes, G.-J. Classical Trajectory Study of the HOCO System Using a New Interpolated ab initio Potential Energy Surface, Chem. Phys. Lett. 2004, 393(1-3), 236-244.

27 Li, J.; Wang, Y.; Jiang, B.; Ma, J.; Dawes, R.; Xie, D.; Bowman, J. M.; Guo, H. Communication: A Chemically Accurate Global Potential Energy Surface for the $\mathrm{HO}+$ $\mathrm{CO} \rightarrow \mathrm{H}+\mathrm{CO}_{2}$ Reaction, J. Chem. Phys. 2012, 136(4), 041103.

28 Kudla, K.; Schatz, G. C.; Wagner, A. F. A Quasiclassical Trajectory Study of the $\mathrm{OH}+\mathrm{CO}$ Reaction, J. Chem. Phys. 1991, 95(3), 1635-1647.

29 Bradley, K. S.; Schatz, G. C. A Quasiclassical Trajectory Study of $\mathrm{H}+\mathrm{CO}_{2}$ : Angular and Translational Distributions, and $\mathrm{OH}$ Angular Momentum Alignment, J. Chem. Phys. 1997, 106(20), 8464-8472.

30 Zhang, D. H.; Zhang, J. Z. H. Quantum Calculations of Reaction Probabilities for $\mathrm{HO}+\mathrm{CO} \rightarrow \mathrm{H}+\mathrm{CO}_{2}$ and Bound States of HOCO, J. Chem. Phys. 1995, 103(15), 6512-6519.

31 Bowman, J. M.; Christoffel, K.; Weinberg, G. Calculations of Low-Lying Vibrational States of cis- and trans-HOCO, J. Mol. Struct. (THEOCHEM) 1999, 461-462(0), 71-77.

32 Lehoucq, R.; Gray, S.; Zhang, D.-H.; Light, J. Vibrational Eigenstates of Four-Atom Molecules: A Parallel Strategy Employing the Implicitly Restarted Lanczos Method, Computer Phys. Commun. 1998, 109(1), 15-25.

33 Mladenović, M. Rovibrational Hamiltonians for General Polyatomic Molecules in Spherical Polar Parametrization. I. Orthogonal Representations, J. Chem. Phys. 2000, 112(3), 1070-1081.

34 Botschwina, P. Accurate Equilibrium Structures for Small Polyatomic Molecules, Radicals and Carbenes, Mol. Phys. 2005, 103(10), 1441-1460. 
35 Fortenberry, R. C.; Huang, X.; Francisco, J. S.; Crawford, T. D.; Lee, T. J. The trans-HOCO Radical: Quartic Force Fields, Vibrational Frequencies, and Spectroscopic Constants, J. Chem. Phys. 2011, 135(13), 134301.

${ }^{36}$ Fortenberry, R. C.; Huang, X.; Francisco, J. S.; Crawford, T. D.; Lee, T. J. Vibrational Frequencies and Spectroscopic Constants from Quartic Force Fields for cis-HOCO: The Radical and the Anion, J. Chem. Phys. 2011, 135(21), 214303.

37 Huang, X.; Fortenberry, R. C.; Wang, Y.; Francisco, J. S.; Crawford, T. D.; Bowman, J. M.; Lee, T. J. Dipole Surface and Infrared Intensities for the cis- and trans-HOCO and DOCO Radicals, J. Phys. Chem. A, Articles ASAP (As Soon As Publishable) 2013, DOI: 10.1021/jp3102546.

38 Wang, Y.; Carter, S.; Bowman, J. M. Variational Calculations of Vibrational Energies and IR Spectra of trans- and cis-HOCO Using New ab Initio Potential Energy and Dipole Moment Surfaces, J. Phys. Chem. A, Articles ASAP (As Soon As Publishable) 2013, DOI: 10.1021/jp309911w.

39 Hampel, C.; Peterson, K. A.; Werner, H.-J. A Comparison of the Efficiency and Accuracy of the Quadratic Configuration Interaction (QCISD), Coupled Cluster (CCSD), and Brueckner Coupled Cluster (BCCD) Methods, Chem. Phys. Lett. 1992, 190(1-2), 1-12.

40 Deegan, M. J.; Knowles, P. J. Perturbative Corrections to Account for Triple Excitations in Closed and Open Shell Coupled Cluster Theories, Chem. Phys. Lett. 1994, 227(3), 321-326.

${ }^{41}$ Knowles, P. J.; Hampel, C.; Werner, H.-J. Coupled Cluster Theory for High Spin, Open Shell Reference Wave Functions, J. Chem. Phys. 1993, 99(7), 5219-5227.

${ }^{42}$ MOLPRO, a package of ab initio programs. Werner, H.J.; Knowles, P. J.; Knizia, G.; Manby, F. R.; Schütz, M.; Celani, P.; Korona, T.; Lindh, R.; Mitrushenkov, A.; Rauhut, G.; et al. see http://www.molpro.net.

43 Meyer, W.; Botschwina, P.; Burton, P. Ab Initio Calculation of Near-Equilibrium Potential and Multipole Moment Surfaces and Vibrational Frequencies of $\mathrm{H}_{3}^{+}$and Its Isotopomers, J. Chem. Phys. 1986, 84(2), 891-900.

44 Press, W. H.; Flannery, B. P.; Teukolsky, S. A.; Vetterling, W. T. Numerical Recipes; Cambridge University Press: Cambridge, 1986.

${ }^{45}$ Krekeler, C. Theoretische Untersuchungen am Radikal $\mathrm{HOCO}$ und dem Anion $\mathrm{HCO}_{2}^{-}$, Diplomarbeit, GeorgAugust-Universität zu Göttingen, Institut für Physikalische Chemie, 2004.

46 Mladenović, M.; Botschwina, P.; Puzzarini, C. SixDimensional Potential Energy Surface and Rovibrational Energies of the HCCN Radical in the Ground Electronic State, J. Phys. Chem. A 2006, 110(16), 5520-5529.

47 Mladenović, M. Discrete Variable Approaches to Tetratomic Molecules: Part I: DVR(6) and DVR(3)+DGB Methods, Spectrochim. Acta, Part A 2002, 58(4), 795-807.

48 Mladenović, M. Efficient Calculation of Rovibrational Energy Levels of General Tetratomic Molecules by Pointwise Representation Methods, In Rollnik, H., Wolf, D., Eds., NIC Symposium 2001, Vol. 9 of NIC Series, pages 85-94, Jülich, 2002. John von Neumann Institite for Computing.

49 Forney, D.; Jacox, M. E.; Thompson, W. E. Infrared Spectra of trans-HOCO, $\mathrm{HCOOH}^{+}$, and $\mathrm{HCO}_{2}^{-}$Trapped in Solid Neon, J. Chem. Phys. 2003, 119(20), 10814-10823.

50 Mielke, Z.; Olbert-Majkut, A.; Tokhadze, K. G. Photolysis of the OC...HONO Complex in Low Temperature
Matrices: Infrared Detection and Ab Initio Calculations of Nitrosoformic Acid, HOC(O)NO, J. Chem. Phys. 2003, 118(3), 1364-1377.

51 Mladenović, M. Vibrational Calculation for the HOCO Radical and the cis-HOCO Anion, J. Chem. Phys. 2012, $137(1), 014306$.

52 Mladenović, M. On Intervector Angle Descriptions and Their Numerical Implementation for Solving Molecular Problems, J. Chem. Phys. 2003, 119, 11513-11525.

53 Mladenović, M.; Elhiyani, M.; Lewerenz, M. Quasilinearity in Tetratomic Molecules: an Ab Initio Study of the CHNO Family, J. Chem. Phys. 2009, 130, 154109.

54 Carter, S.; Murrell, J. N. Analytical Two-Valued Potential Energy Functions for the Ground State Surfaces of $\mathrm{CO}_{2}\left(\widetilde{X}^{1} \Sigma_{g}^{+}\right)$and $\mathrm{CS}_{2}\left(\widetilde{X}^{1} \Sigma_{g}^{+}\right)$, Croat. Chem. Acta 1984, $57,355$.

55 Mladenović, M.; Schmatz, S. Theoretical Study of the Rovibrational Energy Spectrum and the Numbers and Densities of Bound Vibrational States for the System $\mathrm{HCO}^{+} / \mathrm{HOC}^{+}$, J. Chem. Phys. 1998, 109, 4456-4470.

56 Mladenović, M.; Botschwina, P.; Sebald, P.; Carter, S. A Theoretical Study of the Acetylide Anion, $\mathrm{HCC}^{-}$, Theo. Chem. Acc. 1998, 100, 134-146. 


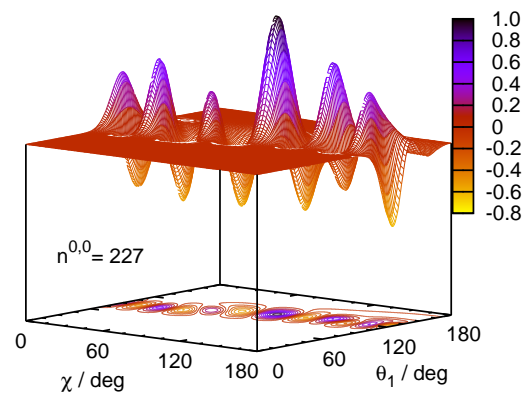

